\title{
Comprehensive study of ULF upstream waves observed in the topside ionosphere by CHAMP and on the ground
}

\author{
B. Heilig ${ }^{1,2}$, H. Lühr ${ }^{1}$, and M. Rother ${ }^{1}$ \\ ${ }^{1}$ GeoForschungsZentrum Potsdam, Telegrafenberg, 1473 Potsdam, Germany \\ ${ }^{2}$ Tihany Geophysical Observatory, Eötvös Loránd Geophysical Institute, Kossuth u. 91., 8237 Tihany, Hungary
}

Received: 11 October 2006 - Revised: 9 February 2007 - Accepted: 12 February 2007 - Published: 29 March 2007

\begin{abstract}
Based on magnetic field measurements from the satellite CHAMP, a detailed picture could be obtained of the upstream wave (UW) distribution in the topside ionosphere. The low, near-polar orbit of CHAMP, covering all local times, allows the global distribution of this type of pulsation to be revealed. The observations from space are compared to recordings of the ground-based MM100 meridional array covering the latitude range $66^{\circ}$ to $42^{\circ}$ in magnetic coordinates. UWs show up very clearly in the compressional component of the satellite magnetic field data, whereas on the ground, their signature is found in the $\mathrm{H}$ component, but it is mixed with oscillations from field line resonant pulsations. Here we first introduce a procedure for an automated detection of UW signatures, both in ground and space data. Then a statistical analysis is presented of UW pulsations recorded during a 132-day period, centred on the autumn 2001 equinox. Observations in the top-side ionosphere reveal a clear latitudinal distribution of the amplitudes. Largest signals are observed at the equator. Minima show up at about $40^{\circ}$ latitude. The coherence between ground and satellite wave signatures is high over wide latitude and longitude ranges. We make suggestions about the entry mechanism of UWs from the foreshock region into the magnetosphere. The clear UW signature in satellite recordings between $-60^{\circ}$ and $60^{\circ}$ latitude allows for detailed investigations of the dependence on solar wind conditions. We test the control of solar wind speed, interplanetary magnetic field strength and cone angle on UWs. For the first time, it is possible to derive details of the Doppler-shift effect by modifying the UW frequency from direct observations. The results reconcile foreshock wave generation predictions with near-Earth observations.
\end{abstract}

Keywords. Magnetospheric physics (Magnetopause, cusp, boundary layers; MHD waves and instabilities; Solar windmagnetosphere interactions)

Correspondence to: $\mathrm{B}$. Heilig

(heilig@elgi.hu)

\section{Introduction}

The ultra low frequency (ULF) upstream waves (UWs) with typical periods of 20-30 s, are waves generated in the Earth's foreshock region. These waves are driven by a wave-particle interaction between the backscattered ions (mainly protons), gyro-rotating around the interplanetary magnetic field (IMF) and the fast magnetosonic waves propagating upstream along the IMF (Barnes, 1970; Gary, 1978, 1991; Yumoto et al., 1984; Le and Russell, 1994; Krauss-Varban, 1994). This process is called the ion-cyclotron or anomalous Doppler-shifted ion/ion resonant instability. As a result of this interaction the frequency of the generated UW is directly proportional to the IMF strength. The key parameter governing the efficiency of the instability is the so-called cone angle, defined as the angle between the Sun-Earth line and the direction of the IMF. The ion-cyclotron instability near the subsolar point of the bow shock is more effective when the IMF is aligned with the solar wind flow, i.e. when the cone angle is small. In this area the bow shock is close to perpendicular to the solar wind flow and so the backscattered ions have an initial velocity almost parallel to the Sun-Earth line. A zero cone angle (Russell et al., 1983; Yumoto et al., 1984; Greenstadt and Russell, 1994), or a small but not zero angle $\left(\sim 30^{\circ}\right)$ between the stream of backscattered ions and the IMF is thought to be the most favourable condition for UW generation (Kovner et al., 1976; Verô and Holló, 1978) and UW transfer through the magnetosheath (Russell et al., 1983). The resulting waves propagate upstream, but since the solar wind is super Alfvénic, i.e. the solar wind speed is usually larger than the phase speed of Alfvén waves in the plasma frame, UWs are swept back to the magnetosphere by the solar wind. These Doppler-shifted waves become left-hand polarized in the spacecraft frame (Yumoto et al., 1984). The efficiency of the wave transfer process (Popatov and Mazur, 1994) and the penetration of UWs through the magnetopause (Kovner et al., 1976), i.e. the power of these waves in the magnetosphere should also depend on the solar wind speed. Under appropriate conditions, the UWs are able to move

Published by Copernicus GmbH on behalf of the European Geosciences Union. 
through the magnetosheath (Russell et al., 1983), enter the magnetosphere near the subsolar point of the magnetopause (Engebretson et al., 1991b; Krauss-Varban, 1994), propagate across the magnetosphere, enter the ionosphere and reach the ground as geomagnetic pulsations (Yumoto et al., 1984).

The connection between the solar wind parameters and the properties of dayside geomagnetic pulsations was first realized in the 1960s. Saito (1964) showed that the amplitude of Pc3s (continuous type pulsations with $10-45 \mathrm{~s}$ period) strongly correlates with the solar wind speed. His work was followed by others (Singer et al., 1977; Wolfe, 1980; Wolfe et al., 1985) with similar conclusions. Yedidia et al. (1991), who compared a year-long record of ground-based daily and hourly mean pulsation activity with solar wind speed, found that the correlation coefficient has a maximum value $(0.86)$ at the 25 -s period pulsations, close to the average upstream period. The first indication of a possible effect of the IMF direction on the occurrence rate of Pc3s was published by Bol'shakova and Troitskaya (1968). According to their results and subsequent studies (PlyasovaBakunina, 1972; Greenstadt and Olson, 1977; Wolfe, 1980; Russell et al., 1983; Yumoto et al., 1985), dayside Pc3s are generally observed on the ground when the cone angle is smaller than $\sim 50^{\circ}$. Some years later, Troitskaya et al. (1971) indicated the existence of a strong correlation between the period of $\mathrm{Pc} 3$ and $\mathrm{Pc} 4$ ( $\mathrm{Pc} 4$ : continuous type pulsations with $45-150 \mathrm{~s}$ period) pulsations and the magnitude of IMF. Series of later studies (Gul'elmi et al., 1973; Verô and Holló, 1978; Verô, 1980; Green et al., 1983) confirmed these results (for additional references see Verô, 1986; Troitskaya, 1994; Hughes, 1994).

The same relationships proved to be valid even for foreshock (Russell and Hoppe, 1981; Le and Russell, 1994) and magnetospheric observations (Yumoto and Saito, 1983; Yumoto et al., 1984). By the mid 1980s it became clear that the dayside pulsation activity had at least partially its origin in the upstream solar wind.

Although there is a general agreement on the generation mechanism of ULF upstream waves in the foreshock region and their presence in the magnetosphere and on the ground, it is still an open question as to what is the dominant channel through which these waves reach the ground. Direct propagation of compressional waves through the magnetosheath and the magnetosphere (e.g. Russell et al., 1983; Yumoto et al., 1984) or coupling of these propagating compressional waves to an evanescent mode at the plasmapause (Kim and Takahashi, 1999) were also proposed. Furthermore, direct penetration at the cusp (Troitskaya and Bol'shakova, 1984) and transfer to the dayside inner magnetosphere via the ionospheric transistor effect has also been suggested (Engebretson et al., 1991a) see Olson and Fraser, 1994, for a review. Recently, Tanaka et al. (2004) applied the ionospheric current model developed for geomagnetic storm theory (Araki, 1994), to explain the longitudinal phase and amplitude structure of Pc3 pulsations near the magnetic equator.
As another exogenous source mechanisms for dayside Pc3-4 pulsations, the Kelvin-Helmholtz instability (KHI) was introduced into pulsation research at the beginning (Dungey, 1954; Chen and Hasegawa, 1974; Southwood, 1974; Hughes et al., 1978; Olson and Rostoker, 1978). Other suggested alternative or additional endogenous sources are the magnetospheric or plasmaspheric compressional cavity mode (Kivelson and Southwood, 1985, 1986; Takahashi and Anderson, 1992; Kim and Takahashi, 1999; Waters et al., 2002), and later the waveguide mode was introduced (Samson et al., 1992; Wright, 1994) (for reviews see Hughes, 1994, and Samson and Rankin, 1994). In the case of endogenous origin there is a further question rarely raised as to where is the origin of the wave energy?

So far, we did not mention the field line resonance (FLR), which is the most frequent phenomenon of dayside Pc3 pulsations. Similar to the aforementioned cavity resonance, it forms an endogenous group of magnetospheric Pc3-4 pulsations. The most striking property of this resonance is its latitude dependence, while the frequency of pulsations associated with UWs, as well as the frequency of cavity resonances, are thought to be basically independent of latitude. The theory of excitation of toroidal mode FLRs by a compressional driver was developed independently by Chen and Hasegawa (1974), and Southwood (1974). Whenever the compressional activity is present in the magnetosphere, the field lines with the eigenfrequencies that are covered by the source spectrum are excited. This means that the source of dayside Pc3-4 activity is at the same time the source of the FLR activity. Correspondingly, first the KHI, later the UW and recently the cavity resonance were thought to be the prime driver of the dayside FLRs.

There are only a few studies published on the compressional wave activity in the inner magnetosphere, in spite of the fact that many satellites carried out magnetic field measurements below the $1000-\mathrm{km}$ altitude. The reason is that satellites in the strong geomagnetic field yielded usually poor resolution due to the limitation in digitization depth (AMPTE CCE had a $8 \mathrm{nT}$ digitization step at perigee, while the resolution of UARS magnetometer was $2 \mathrm{nT}$ at $\sim 580 \mathrm{~km}$ altitude (Takahashi et al., 1999)). So far, it was impossible to extract events having a fraction of $\mathrm{nT}$ amplitudes from the magnetic measurements.

Kim et al. (1998) were the first to investigate a compressional event at $\mathrm{L}<6$. They demonstrated high coherence between the compressional component observed on the morningside (at $\mathrm{L}=3.5-5.3$ ) by AMPTE CCE and an $\mathrm{H}-$ component Pc3 event observed at a low-latitude $(\mathrm{L}=1.25)$ ground station. The event was interpreted as a global plasmaspheric cavity mode. This investigation was later widened into a statistical analysis of compressional Pc3-4 pulsations at $\mathrm{L}=2-3$ in the dayside magnetosphere (Kim and Takahashi, 1999). It was found that the amplitude and phase relationship between the poloidal components, the cross-phase between the compressional component and the $\mathrm{H}$-component at 
Kakioka ( $\mathrm{L}=1.25$ ) are consistent with the standing (cavity) or evanescent mode coupled to inward propagating UWs. They also stated that a further study is required to distinguish between the cavity and evanescent modes.

So far, little attention was paid to the role of UWs in the generation of compressional Pc3-4 pulsations in the inner magnetosphere. Kim and Takahashi (1999) identified only one event in a two-year data set that may be related to an upstream source. Unfortunately, due to the lack of interplanetary data for the overwhelming part of their events, they could not clarify the role of UWs. However, the rather narrow frequency distribution $(10-30 \mathrm{mHz})$ of the 63 events with a median frequency of $20 \mathrm{mHz}$ actually makes us believe that the direct source of these pulsations was more likely cavity resonances rather than UWs.

In this paper we study compressional pulsations observed at a low Earth orbit (LEO) by the CHAMP satellite which at $350-450 \mathrm{~km}$ altitude, visits almost all latitudes and local times during a four-month period, owing to its nearpolar orbit. With the help of CHAMP's high resolution, low noise fluxgate magnetometer measurements, we first map the global distribution of dayside compressional ULF wave activity.

The UWs can be found in the compressional component of the magnetospheric magnetic field (Yumoto et al., 1984; Takahashi et al., 1994). In the magnetosphere, where the compressional (field-aligned) component dominates over the transverse components, the wave signature can well be derived from the total field variations (Jadhav et al., 2001). Magnetospheric FLRs are mostly toroidal resonances which can be observed in the transverse components. One of the advantages of using LEO satellites for UW study is that FLR and UW activity manifest themselves in different components above the ionosphere (Yumoto et al., 1984; Takahashi et al., 1994), so they can easily be separated. In contrast, on the ground, due to the ionospheric screening and the $90^{\circ}$ rotation, both activities show up in the $\mathrm{H}$ (magnetic north) component (Nishida, 1978; Takahashi et al., 1994). That is why a more sophisticated procedure is needed to identify UW events from ground-based measurements. To our knowledge, no automated algorithm has been developed up to now for this purpose.

In the present paper we concentrate on the compressional ULF wave activity occurring on the dayside within the $\pm 60^{\circ}$ magnetic latitude range. We introduce a simple algorithm developed for selecting Pc3 events, assumed to be UW related pulsation, from space, as well as from ground magnetometer signals. With the help of the large data sets built up by the automated algorithm, we try to revisit some of the key issues of UW related pulsation theory. Our initial step is mapping the global distribution of compressional power in the Pc3 band at LEO altitudes. Then we determine the ground-space coherence of Pc pulsations and outline the low- and mid-latitude channels through which these waves reach the topside ionosphere. The amount and quality of the data makes it also possible to analyse the dependence of the properties of UW related pulsations on solar wind conditions in much more detail than before.

\section{Instrumentation and data}

\subsection{Satellite data}

The CHAMP satellite was launched on 15 July 2000 into an almost circular, near polar (inclination $87.3^{\circ}$ ) orbit, with an initial altitude of $454 \mathrm{~km}$ which has changed to $\sim 350 \mathrm{~km}$ after 5 years. An advantage of this orbit is the local time (LT) variation of the satellite's ground track which makes it possible to investigate the LT dependence of various phenomena. For full local time coverage in statistical studies data with 132 days have to be used. In the present study we chose the period from 1 August to 11 December 2001, which is centered around the autumn equinox, to minimize the possible effects of seasonal hemispheric asymmetry on our results.

The satellite data used in this study are the preprocessed (level 2) fluxgate vector magnetometer data from CHAMP (product identifier CH-ME-2-FGM-FGM). These are publicly available through the CHAMP Information System and Data Center (http://isdc.gfz-potsdam.de/champ/). This instrument samples the magnetic field at a rate of $50 \mathrm{~Hz}$ with a resolution of $0.1 \mathrm{nT}$. In the standard processing the data are averaged to $1-\mathrm{Hz}$ samples. The fluxgate measurements are regularly calibrated against an absolute scalar (Overhauser) magnetometer to ensure continuous accuracy over the multiyears mission. With CHAMP it is possible, for the first time, to study the small amplitude UW-related events (Sutcliffe and Lühr, 2003; Vellante et al., 2004). For the statistical analysis of compressional waves observed by CHAMP we used the total field values calculated from the low noise fluxgate vector measurements. In general, it is desirable to also use the field vector components in pulsation studies, as was done in the two above-mentioned papers. Unfortunately, the standard transformation of the satellite measurements into an Earth-oriented frame adds undesirable attitude noise to the data. Generating clean vector data requires a lot of manual intervention. We demonstrate in this study that a lot of UW features can be derived from the total field signature.

To avoid a false interpretation of spatial structures as pulsation signals, resulting from the fast moving satellite through the ambient field, the field model POMME 2.5 (Potsdam Magnetic Model of the Earth) (Maus et al., 2005) was subtracted from the measurements. This model includes the main field, the field of the ring current, largescale magnetospheric fields, and the crustal anomalies up to spherical harmonic degree/order 90 (Maus et al., 2006). In particular, the removal of the last term was shown to be important for pulsation studies (Sutcliffe and Lühr, 2003; Vellante et al., 2004). POMME can be downloaded from the GFZ website (http://www.gfz-potsdam.de/ 
Table 1. Stations of the MM100 Pulsation Recording Array.

\begin{tabular}{|c|c|c|c|c|c|c|c|}
\hline \multirow{2}{*}{$\begin{array}{l}\text { Station } \\
\text { Name }\end{array}$} & \multirow{2}{*}{$\begin{array}{l}\text { Operating } \\
\text { Institute }\end{array}$} & \multirow{2}{*}{$\begin{array}{l}\text { IAGA } \\
\text { Code }\end{array}$} & \multicolumn{2}{|c|}{ Geographic coordinates } & \multicolumn{2}{|c|}{ AACGM coordinates (2001) } & \multirow{2}{*}{$\begin{array}{l}\text { McIllwain } \\
\text { L shell }\end{array}$} \\
\hline & & & Latitude N & Longitude E & Latitude N & Longitude E & \\
\hline Kilpisjärvi & IMAGE & KIL & $69.02^{\circ}$ & $20.79^{\circ}$ & $66.10^{\circ}$ & $104.00^{\circ}$ & 6.09 \\
\hline Sodankylä & IMAGE & SOD & $67.37^{\circ}$ & $26.63^{\circ}$ & $64.16^{\circ}$ & $107.46^{\circ}$ & 5.26 \\
\hline Hankasalmi & IMAGE & HAN & $62.30^{\circ}$ & $26.65^{\circ}$ & $59.01^{\circ}$ & $104.78^{\circ}$ & 3.77 \\
\hline Nurmijärvi & IMAGE & NUR & $60.52^{\circ}$ & $24.65^{\circ}$ & $57.23^{\circ}$ & $102.34^{\circ}$ & 3.41 \\
\hline Tartu & IMAGE & TAR & $58.26^{\circ}$ & $26.46^{\circ}$ & $54.48^{\circ}$ & $103.04^{\circ}$ & 3.01 \\
\hline Belsk & PAS & BEL & $51.83^{\circ}$ & $20.80^{\circ}$ & $48.01^{\circ}$ & $96.15^{\circ}$ & 2.23 \\
\hline Hurbanovo & SAS & HRB & $47.87^{\circ}$ & $18.18^{\circ}$ & $43.56^{\circ}$ & $92.86^{\circ}$ & 1.90 \\
\hline Nagycenk & ELGI-USGS & NCK & $47.63^{\circ}$ & $16.72^{\circ}$ & $43.27^{\circ}$ & $91.53^{\circ}$ & 1.89 \\
\hline Farkasfa & ELGI-USGS & FKF & $46.91^{\circ}$ & $16.31^{\circ}$ & $42.43^{\circ}$ & $91.00^{\circ}$ & 1.84 \\
\hline Tihany & ELGI-USGS & THY & $46.90^{\circ}$ & $17.89^{\circ}$ & $42.44^{\circ}$ & $92.39^{\circ}$ & 1.84 \\
\hline
\end{tabular}

pb2/pb23/SatMag/model.html). However, one has to be aware that there are other magnetic signature associated, for example, with plasma density fluctuations called equatorial spread-F (e.g. Stolle et al., 2006) or F region current systems, which are not taken into account (Lühr et al., 2002). They can disturb the estimation of pulsation activity.

\subsection{MM100 ground magnetometer array}

MM100 is the synonym for a quasi-meridional magnetometer array established in September, 2001 for pulsation study (Table 1). The array consists of Finnish, Estonish, Polish, Slovak and Hungarian stations from high to mid latitudes ( $\mathrm{L}=6.09$ to 1.84 ). The Finnish and Estonish sites belong to the IMAGE (Lühr et al., 1998) array. The Polish and Slovak Observatories are maintained by the national Academies of Sciences. The Hungarian stations were installed in 1999 as a result of a common effort of the USGS and the Eötvös Loránd Geophysical Institute (ELGI) in Hungary. The equipments used are high resolution fluxgate or torsion photoelectric magnetometers, both with GPS synchronized timing and sampled at $1 \mathrm{~Hz}$. All ground magnetometer data were transformed into the magnetic field oriented HDZ system before analysis.

2.3 ACE solar wind (SWEPAM) and interplanetary magnetic field (MFI) data

The interplanetary magnetic field (IMF) and solar wind plasma data used in this study are from the Magnetic Field Experiment (MFI) and Solar Wind Electron Proton Alpha Monitor (SWEPAM) on board the ACE (Advanced Composition Explorer) spacecraft. The data are used in the geocentric solar magnetospheric (GSM) system with a time resolution of $64 \mathrm{~s}$. The calibrated level-2 data are available at the ACE Science Center public domain. During the period of interest, ACE was orbiting around the Lagrange point, L1, some $230 R_{E}$ in front of the Earth. The solar wind needs approximately an hour, depending, first of all, on the ac- tual solar wind speed to travel the distance between L1 and the magnetosphere. Before comparing solar wind conditions with our pulsation observation events, we delayed the ACE time series by the travel time calculated from the solar wind parameters. Data were smoothed by filtering, shorter data gaps were filled by linear interpolation.

\subsection{Data processing}

The expected frequencies of the UW events being investigated are some tens of $\mathrm{mHz}$. For the estimation of these frequencies a few $\mathrm{mHz}$ resolution is needed. Using the Fourier transform this would require 15-20 min intervals of data. However, since the CHAMP is moving with a speed of $4^{\circ}$ lat./min, these long intervals corresponding to a $60^{\circ}-80^{\circ}$ latitude range would make it impossible to investigate the spatial structure of these waves. The Morlet wavelet analysis proved to be a suitable tool to overcome this problem, as it has the property to measure all the frequencies on their appropriate scales. The application of wavelet analysis is more adequate even for the rather dynamic signal. The wavelet power spectral density (PSD $-P_{x}$ ) of a signal and the cross-spectral density $\left(\mathrm{CSD}-P^{n}\right)$ of $n$ signals are defined as:

$P_{x}=|\hat{X}(t, s)|^{2}$

$P^{n}=\sqrt[n]{\prod_{i=1}^{n}\left|\hat{X}_{i}(t, s)\right|^{2}}$,

where $\hat{X}(t, s)$ and $\hat{X}_{i}(t, s)$ are the wavelet transform of $x(t)$ and $x_{i}(t)$ time series, $t$ is the time, and $s$ is the wavelet scale.

The processing of UW pulsation, as observed by CHAMP, consists of the following steps: 1) removing the background field using the POMME model; 2) differentiating and bandpass filtering the data $(16-100 \mathrm{mHz})$ using a Butterworth filter; 3) calculating PSD estimates of the compressional (total field) component using a continuous Morlet wavelet transform. This transform yields an estimation of PSD for every 
second; 4) determining the peak power and the mean power of the mean PSD for every minute; 5) deriving the frequency corresponding to the power peak (called peak frequency).

The UW frequency estimation for the ground array follows a similar scenario. The only difference is that we do not consider the variation of the field magnitude but the horizontal component, $\mathrm{H}$. In this case the UW cannot be estimated from data recorded at a single station, but measurements from a meridional chain have to be considered since the signals of the investigated pulsations are strongly masked by local resonance features, i.e. FLRs. Supposing that the UW is coherent over large areas extending up to thousands of kilometres on the ground (Tanaka et al., 1998), this activity should become visible by calculating the CSD of the signals recorded at MM100 stations on different geomagnetic latitudes while weakening latitude dependent resonances. The four steps for UW estimations from the ground meridional array are the same as mentioned above, except for 1) converting all data to HDZ orientation system and 3) calculating CSD estimates of the $\mathrm{H}$ component using a continuous Morlet wavelet transform.

In addition to separate spectral analysis of ground and satellite data, we also computed the ground-space coherence for all the ground stations. The wavelet coherence can be defined as:

$$
R_{x y}(t, s)=\frac{|\langle\hat{X}(t, s) \overline{\hat{Y}(t, s)}\rangle|}{\sqrt{\left\langle|\hat{X}(t, s)|^{2}\right\rangle\left\langle|\hat{Y}(t, s)|^{2}\right\rangle}}
$$

where $<>$ means smoothing, both in time and scale. The smoothing function is designed so that it has a similar footprint as the wavelet used. For the Morlet wavelet applied in our analysis following Grinsted et al. (2004), a running averaging with Gaussian weights in time and a boxcar averaging in scale was used. Because of smoothing the wavelets, the coherence is less well defined, both in time and frequency. The time averaging extends over 6 periods (6T) at every scale, i.e. the length of the running window is $6 \mathrm{~T}$, but the sum of the weights is as high as 0.98 even in the $(-2 \mathrm{~T}, 2 \mathrm{~T})$ interval and 0.77 in $(-1 \mathrm{~T}, 1 \mathrm{~T})$. For a $16-100-\mathrm{mHz}$ signal the window length is between $1-6 \mathrm{~min}$, which means, in spite of the fast movement of CHAMP, the wavelet coherence can still be a useful quantity in a ground-space comparison analysis for yielding local information.

Similarly, the one-minute mean space-ground coherences at CHAMP's peak frequency were computed for all MM100 stations.

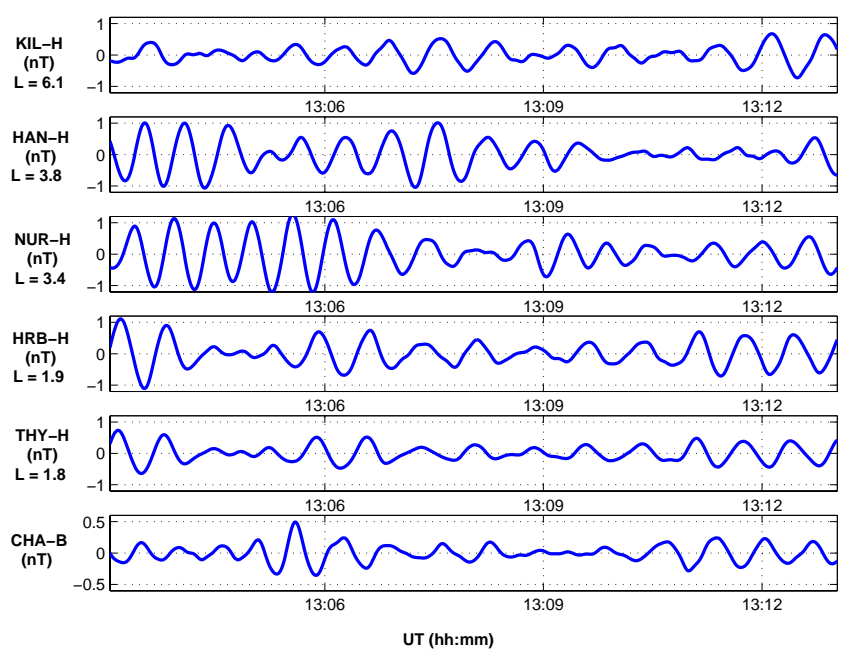

Fig. 1. H-component measured at some stations of the MM100 meridional array (Kilpisjärvi, Hankasalmi, Nurmijärvi, Hurbanovo, Tihany) and the compressional component observed by CHAMP (bottom panel) when passing along $-7^{\circ}$ longitude, from $70^{\circ}$ to $30^{\circ}$ latitude on 2 September 2001 (13:03-13:13 UT).

\section{Observations and interpretation}

\subsection{Ground-satellite comparison}

Figure 1 shows, as an example, pulsations recorded simultaneously on the ground ( $\mathrm{H}$ component) and by the satellite (total field) for a CHAMP pass over Europe. During this pass, on 2 September 2001, CHAMP travelled approximately along the $7^{\circ}$ west meridian from north to south $\left(70^{\circ}\right.$ to $30^{\circ}$ latitude). The first five panels show a 10-min (13:0313:13 UT) long interval of the $\mathrm{H}$-component signals for some MM100 stations (L from 6.09 to 1.84). In the bottom panel the CHAMP compressional component is presented. The most striking similarities can be observed between the midlatitude stations (HRB and THY) and the CHAMP compressional component. We found this observation to be valid for most of the events. The observed phase delay of about $180^{\circ}$ between CHAMP and THY recordings is primarily a consequence of their longitudinal separation $\left(25^{\circ}\right)$, but the space-ground phase difference seems to also depend on local time. A proper interpretation of phase relations should take into account all the possible wave modes reflected in the $\mathrm{H}$-signal on the ground and in the compressional activity in space, as well as the role of the ionosphere. The detailed interpretation is beyond the scope of this study. During the investigated period, the general activity level varies in the same manner and also the dominant frequencies estimated by autocorrelation for the same interval are close to each other (KIL: $24 \mathrm{mHz}$, HAN: $29 \mathrm{mHz}$, NUR: $30 \mathrm{mHz}, \mathrm{HRB}$ : $26 \mathrm{mHz}$, THY: $25 \mathrm{mHz}$, CHAMP: $28 \mathrm{mHz}$ ). On the ground, according to cross-correlation analysis, the signal is delayed poleward. 


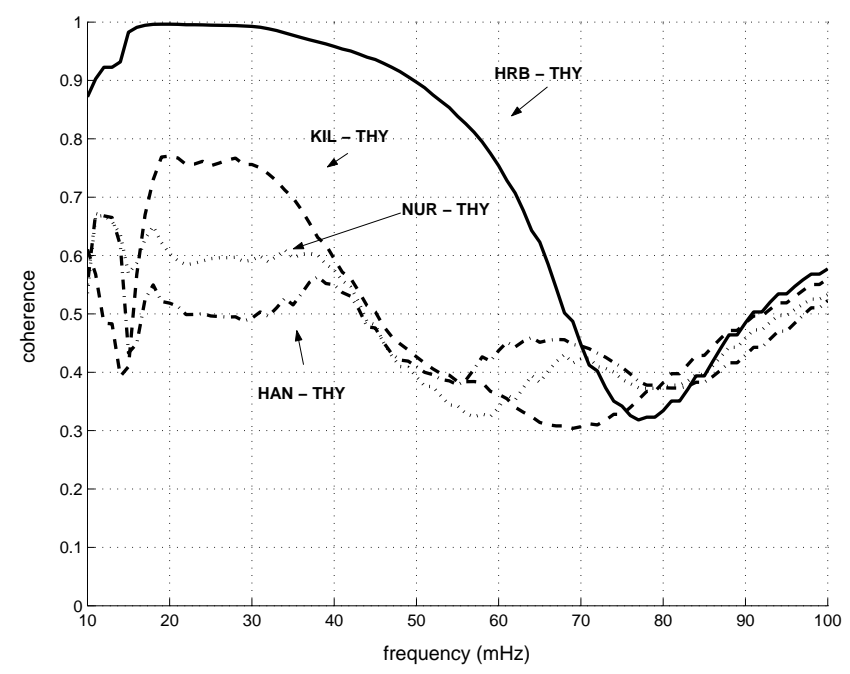

Fig. 2. MM100 interstation signal coherence of $\mathrm{H}$ component for the sample period (same as for Fig. 1)

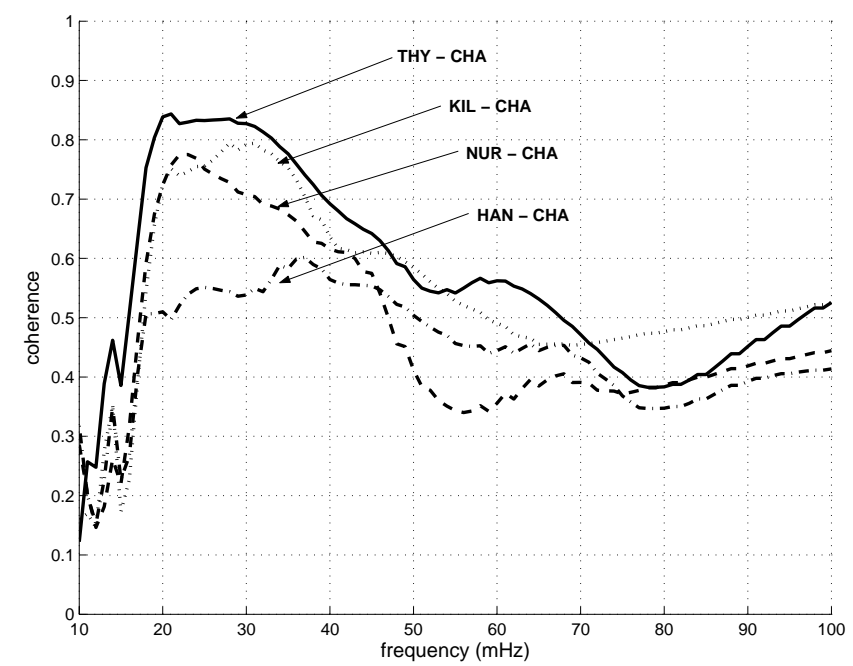

Fig. 3. Ground-to-space signal coherence for the sample period (same as for Fig. 1)

We estimated the coherences between the MM100 stations and THY, used here as the reference station (Fig. 2), and also computed the ground-space coherences (Fig. 3). Both for the ground-ground and the space-ground correlation the coherence decreases the higher the latitude of the considered station, except for KIL, where the coherence is high again. The latitudinal length scale corresponding to a coherence coefficient of $>0.6$ is at least $1500 \mathrm{~km}$ for the investigated event. The pulsations observed on the ground seem to be closely related to the compressional wave activity at about $450-\mathrm{km}$ altitude.

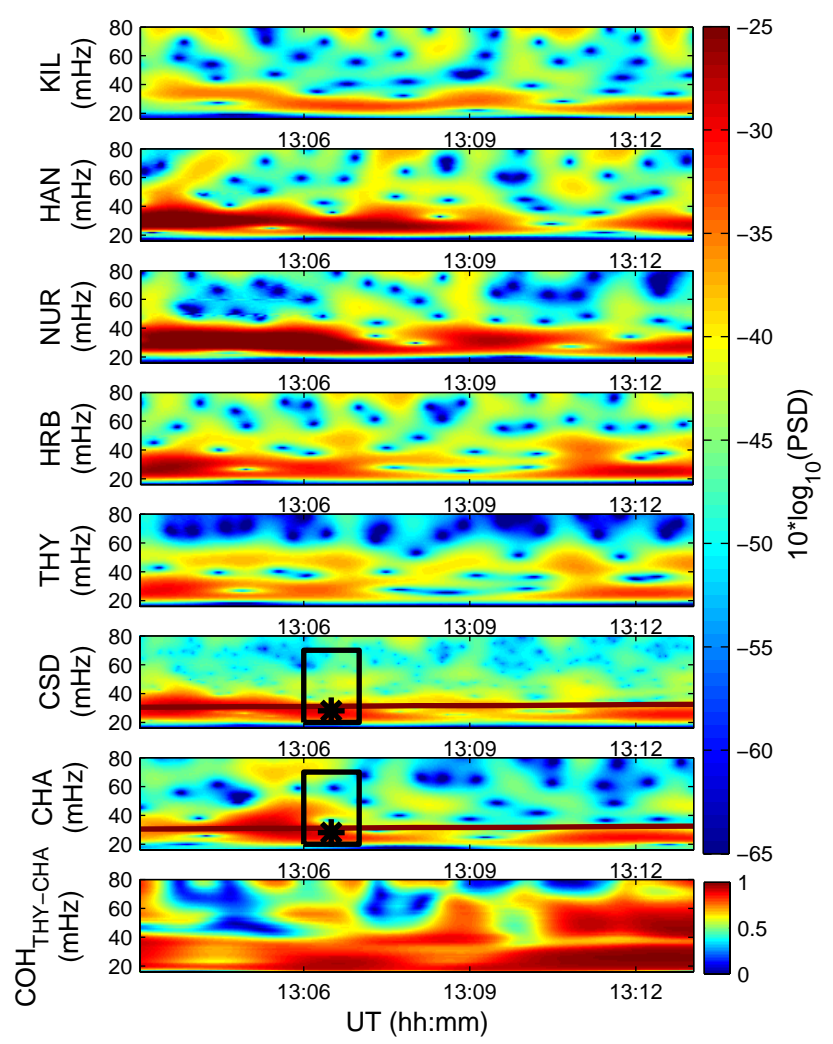

Fig. 4. Morlet-wavelet power spectral density (PSD) estimation of the time differentiated signals presented in Fig. 1. The first five panels represent the PSDs of the differentiated $\mathrm{H}$ component at Kilpisjärvi, Hankasalmi, Nurmijärvi, Hurbanovo and Tihany, respectively; (6th panel) cross-spectral density (CSD) of the five ground signals; (7th panel) PSD of the total field variation at CHAMP. The solid lines in the 6th and 7th panel show the UW frequency estimated from the IMF strength. Rectangles outline the cells from which the mean power is calculated: the black stars mark the frequencies at power peaks; (bottom panel) $\mathrm{CHAMP}^{-\mathrm{B}_{v^{-}}}$ to-THY-H coherence.

In Fig. 4 the continuous Morlet wavelet transforms (PSD) of the same signals are presented. In the first five panels the H-components of five MM100 stations are shown, and in the seventh panel the CHAMP compressional component is depicted. The sixth panel contains the cross-spectral power density (CSD) computed from the MM100 stations. Morlet scales were transformed to frequencies for all PSD and CSD dynamical spectra for simplicity. In the 6th and 7th panel the expected UW frequency is also shown (brown curve), as calculated from the often cited equation, $f_{U W}(\mathrm{mHz})=6 B_{\mathrm{IMF}}$ (nT) (e.g. Yumoto et al., 1984). The bottom panel of Fig. 4 presents the coherence spectrum between the CHAMP and THY signals. In the presented case the IMF cone angle was less than $30^{\circ}$, which is considered to be favourable for UW generation. In the MM100 CSD and CHAMP panels oneminute band-limited $(20-70 \mathrm{mHz})$ cells, black rectangles, are 


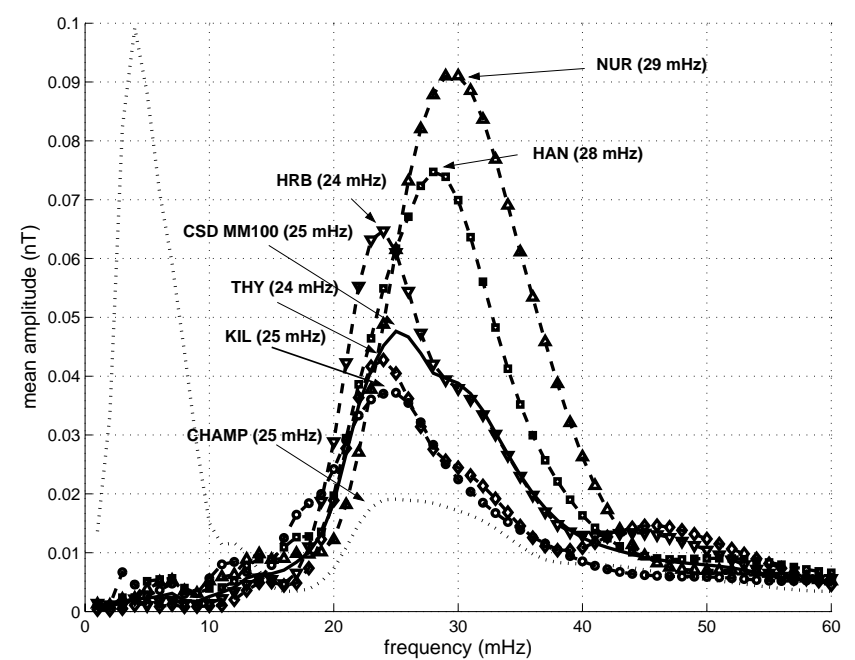

Fig. 5. Mean power spectral density of the time differentiated signals, i.e. time averages of the spectra presented in Fig. 4. The low frequency peak of the CHAMP spectrum is caused by residual crustal anomalies not removed by the POMME model.

also shown. The mean power density values are calculated in such cells. The peak frequency (black stars) is estimated in similar cells, but over a wider $(15-100 \mathrm{mHz})$ band.

In the presented case an enhanced activity can be observed in the $20-40 \mathrm{mHz}$ band at all stations and even onboard CHAMP (Fig. 5.). The CSD of MM100 stations, which is amplifying the common driver signal (here UW) and suppressing the local signal contributions (e.g. resonant response), yields a particularly clear view of this continuous narrowband pulsation activity. In Fig. 5 the average spectra for the same period are presented. The calculated spectral peaks are all close to the expected UW frequency, 30$32 \mathrm{mHz}$, estimated from IMF strength. The signal power is highest at NUR, decaying both poleward and equatorward, and is weakest in the topside ionosphere (CHAMP). The relative half-peak value bandwidth is 0.62 and 0.57 , the $\mathrm{S} / \mathrm{N}$ ratio is $3.8 \mathrm{~dB}$ and $19.6 \mathrm{~dB}$ for the CHAMP and MM100 CSD spectra, respectively.

We note that at HRB, and especially at THY a secondary spectral line can be distinguished near $40-50 \mathrm{mHz}$. It is the weak resonant response of the local magnetic shell at the eigenfrequencies of the local field lines.

From the three properties of this event, namely (1) apparent poleward propagation, (2) highest coherence at lowest latitudes, and (3) highest power near NUR, we conclude that ground signals are caused by waves guided along local field lines driven by an inward propagating UW-related compressional wave as a common source. The compressional wave couples to toroidal mode during its passage through the inhomogeneous magnetosphere. The efficiency of mode coupling depends on the topology of field lines and the ratio of the fre-
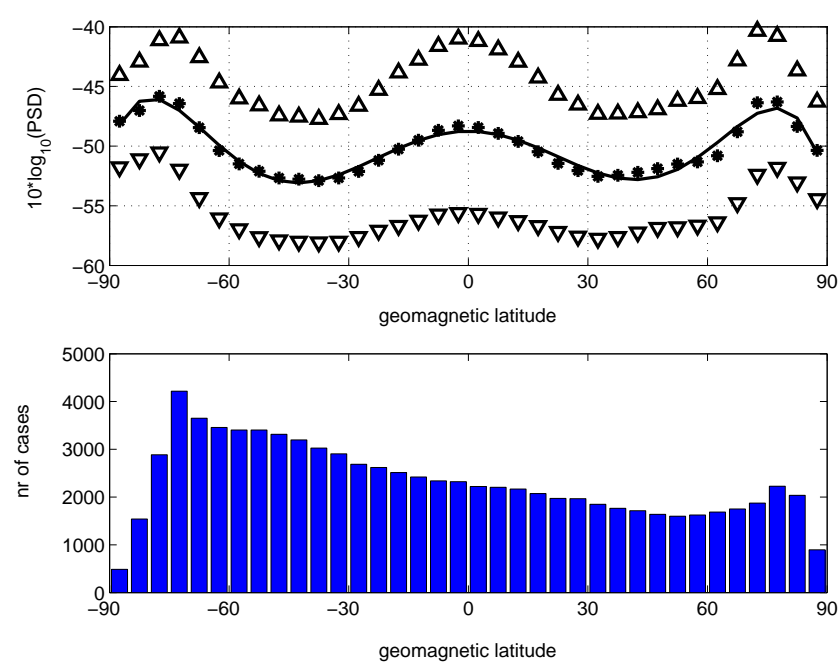

Fig. 6. Mean latitudinal distribution of dayside $\left(\chi<90^{\circ}\right)$ compressional signal power observed by CHAMP between 1 August-11 December 2001. In the top panel the stars represent the mean power distribution, the solid line is a 6th order polynomial fitted to the observations, while the triangles show the observed standard deviations. In the bottom panel the latitudinal distribution of considered spectra is presented.

quency between the compressional wave and the local field line eigenfrequency. It is highest in volumes where the driver frequency matches the local field line eigenfrequency and the wave vector is parallel to ambient field.

Since the field lines mapping to high latitudes are longer than those mapping to mid-latitudes and the Alfvén speed is slower than the incoming fast mode, it takes more time for the guided waves to reach higher latitudes. The travel time of a guided wave between the field line apex and the ionospheric footprint is one-quarter of the local eigenperiod. Using the phase gradient method we obtained the eigenfrequencies $6-7 \mathrm{mHz}$ and $40-45 \mathrm{mHz}$ for the station pairs HANNUR and HRB-THY, respectively. Thus, the difference between the travel times along the two paths is $1 / 4 T_{\mathrm{HAN}-\mathrm{NUR}}-$ $\left(1 / 4 T_{\mathrm{HRB}-\mathrm{THY}}-\Delta L^{*} R_{E} / v_{A}\right)$, where $T_{\mathrm{ST} 1-\mathrm{ST} 2}$ is the eigenperiod of the field line resonance at the ST1-ST2 station pair, $\Delta L * R_{E}$ is the distance between the field line apexes, and $v_{A}$ is the average Alfvén speed in the corresponding region. This gives all together a time difference of 19-28 s. According to the result of the cross-correlation analysis, the delay at NUR with respect to THY is $20 \mathrm{~s}$, a value in good agreement with the above estimate. This scenario can also provide an explanation for the observation that the ground-space coherence is higher for lower latitude ground stations.

\subsection{Latitude dependence of CHAMP compressional power}

For a statistical study of compressional wave power observed by CHAMP, four consecutive months of data were selected 

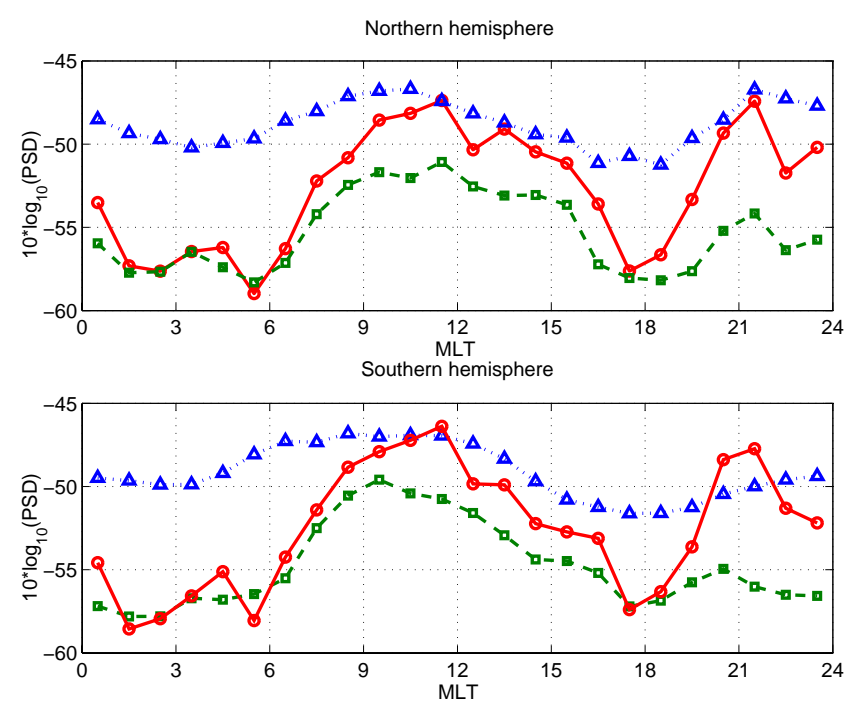

Fig. 7. Local time dependence of normalized compressional signal power observed by CHAMP in different zones. The power is normalized to a $400 \mathrm{~km} / \mathrm{s}$ solar wind speed. In the upper panel triangles/rectangles/circles represent the mean compressional signal power in the $60^{\circ}-90^{\circ} / 30^{\circ}-60^{\circ} / 0^{\circ}-30^{\circ}$ northern latitudinal zones, respectively. The lower panel shows the same for the Southern Hemisphere.

to obtain a full coverage of all local times (1 August 2001-11 December 2001). The average dayside compressional power in the $20-70 \mathrm{mHz}$ band has a peak near the geomagnetic equator and also peaks at high latitudes in a region mapping to the magnetopause (Fig. 6). The latitudinal characteristic is rather symmetrical about the dip-equator for the investigated period around autumn equinox. The peak values at high latitudes and at the equator have similar magnitudes. Minimum values are observed at about $40^{\circ}$ in both hemispheres. Possible reasons for the minima are discussed in the next section.

Takahashi and Anderson (1992), who analysed events in the zone limited by MLAT $= \pm 16^{\circ}$ and $\mathrm{L}=2.5-6.5$, reported the greatest dayside compressional Pc3 amplitudes at the magnetic equator. Jadhav et al. (2001) also observed enhanced amplitudes during daytime at LEO (Ørsted) near the equator.

We relate the peaks at auroral latitudes $\left(75^{\circ}\right.$ mag. lat.) to a different generation process. Their appearance is independent of the solar wind parameters that control the UWs. It is more the merging electric field, $E_{m}$, that correlates well with the activity $\left(E_{m}=v_{s w} \sqrt{B_{y}^{2}+B_{z}^{2}} \sin ^{2}(\theta / 2)\right.$, where $v_{s w}$ is the solar wind speed, $B_{y}$ and $B_{z}$ are the IMF components, $\theta$ is the clock angle all in GSM coordinates). The merging electric field has a close control of FACs on the dayside, as was shown recently, for example, by Wang et al. (2005), based on CHAMP measurements. When interpreting the field variations observed in the auroral regions as spatial structures, one

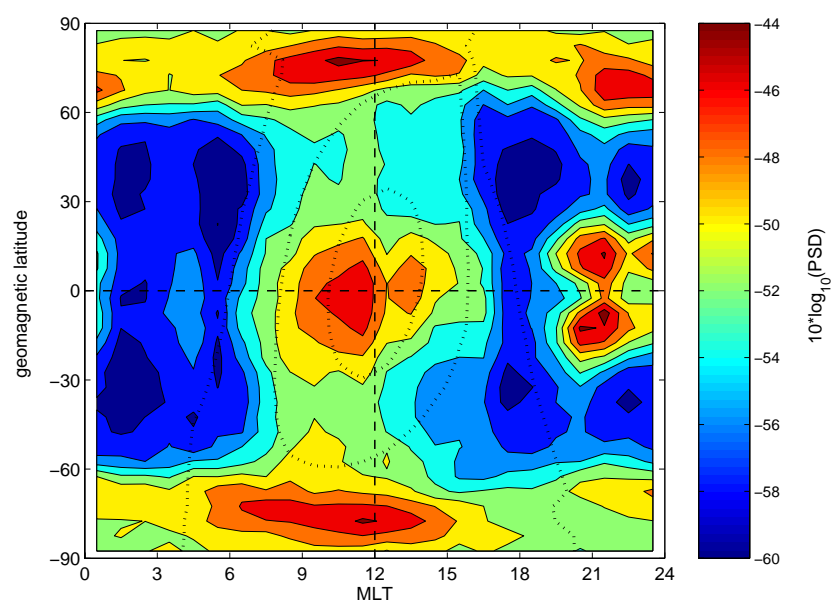

Fig. 8. Magnetic latitude versus magnetic local time distribution of the normalized mean compressional signal power. The power is normalized to a $400 \mathrm{~km} / \mathrm{s}$ solar wind speed. Dotted curves represent the average position of the $90^{\circ}, 60^{\circ}$ and $30^{\circ}$ solar zenith angle isolines.

finds a spectral peak at wavelengths of $100-300 \mathrm{~km}$. This fits well the thickness of major FAC sheets. The signal we observe here probably reflects just the small contribution of the FAC-related magnetic field, $B_{\mathrm{FAC}}$, to the total field reading, $\triangle B=B_{\mathrm{FAC}}^{2} / 2 B$, where $B$ is the total field. Magnetic signatures from $E$ region currents, such as the electrojets, are less likely to play a role, since they have larger wavelengths at the CHAMP altitude.

\subsection{Local time dependence and global distribution of com- pressional power}

The local time distribution at different latitude zones is shown in Fig. 7, where the compressional power was normalized to $400 \mathrm{~km} / \mathrm{s}$ solar wind speed, as explained later in Sect. 3.5. Most pronounced is the variation at low latitudes. Immediately after sunrise (06:00 LT) there is a steep rise in power. It peaks around 11:00 LT. In the afternoon there is a secondary peak at 13:30 LT. By the time of sunset (18:00 LT) the power has reached the night-time level again. Interestingly, there is another increase in power after 19:00 LT, lasting until midnight. This night-time enhancement is present only at low latitudes. The mid-latitude variations are very similar to that previously described, but on a somewhat lower level and without the peak at night. At auroral latitudes the diurnal variation is quite different. First, the signal level is rather high throughout the day and secondly, maxima occur in the pre-noon and pre-midnight sectors. We regard these maxima as being caused by FACs in the polar cusp/cleft and in the substorm region, respectively.

A synoptic perspective of the latitude and local time distribution is given in Fig. 8. From this global view it is evident 


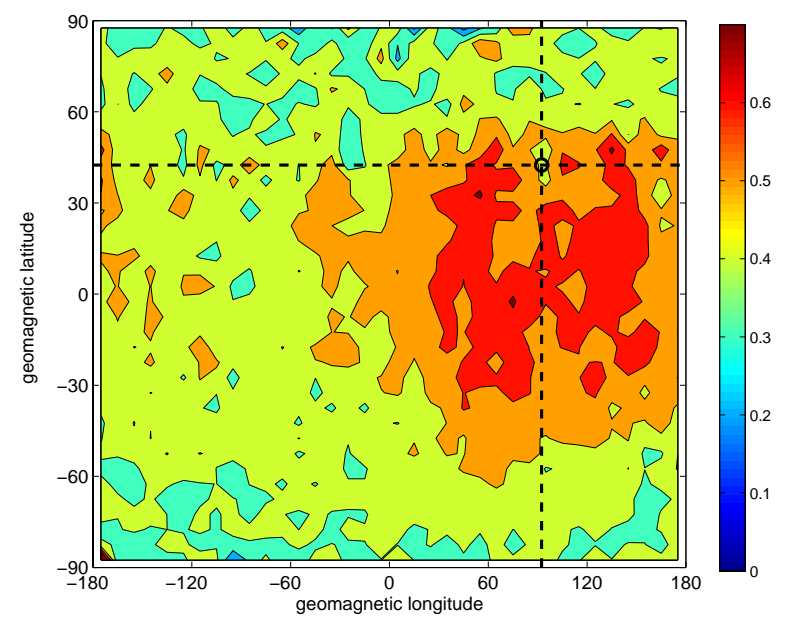

Fig. 9. The global distribution of the coherence at peak power between THY-H and CHAMP compressional waves averaged over the dayside $\left(\chi<90^{\circ}\right)$. The circle and the dotted lines mark the position of THY.

that there are three isolated groups of compressional wave activity in the CHAMP data. We have classified them in the following way: group 1 is confined to the dayside (06:0018:00 LT) within the latitude band $\pm 60^{\circ}$ mag. lat.; group 2 reflects the night time activity. This can clearly be related to the plasma instabilities as part of the equatorial spread $\mathrm{F}$ phenomenon. A detailed description of the magnetic signature associated with the irregularity, as observed by CHAMP, was given by Stolle et al. (2006). Group 3 is confined to high latitudes poleward of $60^{\circ} \mathrm{mag}$. lat. The signal is thus dominated by auroral activity. As a result, we consider only the wave activity within Group 1 as related to UWs and will concentrate, therefore, in subsequent sections on data from this latitude/local time window.

\subsection{Latitude and longitude dependence of space-ground co-} herence

The global distribution of the average dayside space-ground coherence at the peak frequency for the stations Tihany and Nurmijärvi are shown in Figs. 9 and 10. For these figures the samples were binned according to the longitude, to place the emphasis on the high-coherent space-ground coincidences. The investigated period is somewhat shorter (1 September10 December 2001), but the mean date of the selected samples (28 September) is still close to equinox. In case of THY the latitudinal and longitudinal coherence scale lengths are at least $100^{\circ}$ and $180^{\circ}$ (i.e. $11000 \mathrm{~km}$ and $20000 \mathrm{~km}$ ), respectively. The coherence is highest when the satellite is close to the geomagnetic equator, and about $40^{\circ}$ off the magnetic meridian of THY. Along the magnetic meridian of THY the coherence is somewhat lower. This distribution is also in accordance with the UW origin of both the compressional

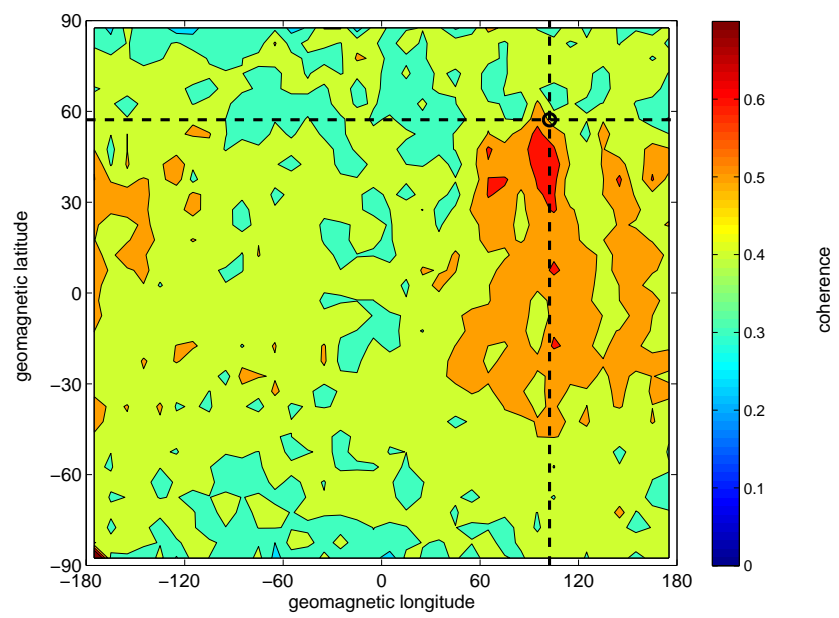

Fig. 10. The same as Fig. 9, but for the coherence between NUR and CHAMP.

activity in the topside ionosphere between $-60^{\circ}$ and $+60^{\circ}$ mag. lat. and 06:00-18:00 MLT.

Conversely, in the case of NUR the coherence is enhanced along the local meridian with a much shorter longitudinal coherence length. The maximum coherence occurs at about $40^{\circ}-45^{\circ} \mathrm{mag}$. lat. This is the latitude range where the coupling between the inward propagating compressional wave and the shear Alfvén mode is supposed to be the strongest. The coupled Alfvén mode travels further along the geomagnetic field lines, as discussed below in Sect. 4. It is possible that the dayside Pc3 activity at NUR reaches the ground via field guided Alfvén waves. The north-south asymmetry in the coherence map may indicate that field guided waves reflected from the conjugate ionosphere are damped during their propagation, or part of their energy is dissipated in the ionosphere. The strong coupling near local noon, on the other hand, can explain the moderate THY-CHAMP coherence along the meridian of THY, and the local minimum around THY, which lies at 42.4 mag. lat., in a latitude range, where the average local FLR frequency $(40-50 \mathrm{mHz})$ is close to the average UW frequency; hence, the coupling is rather effective between the two modes. When the driving UW frequency and the local eigenfrequency differ somewhat, as in the majority of cases, the coherence between them drops significantly.

\subsection{Relation of UWs to SW conditions}

In a second step the events of the Group 1 ranked as candidate UW events were tested against the well-known properties of UWs. The dependence of activity (mean power) on solar wind speed (Fig. 11) and IMF cone angle (Fig. 12) shows up rather clearly, as expected for UWs (Wolfe et al., 1985; Yedidia et al., 1991; Verô and Zieger, 1994). The higher speed solar wind provides a higher power input. From 

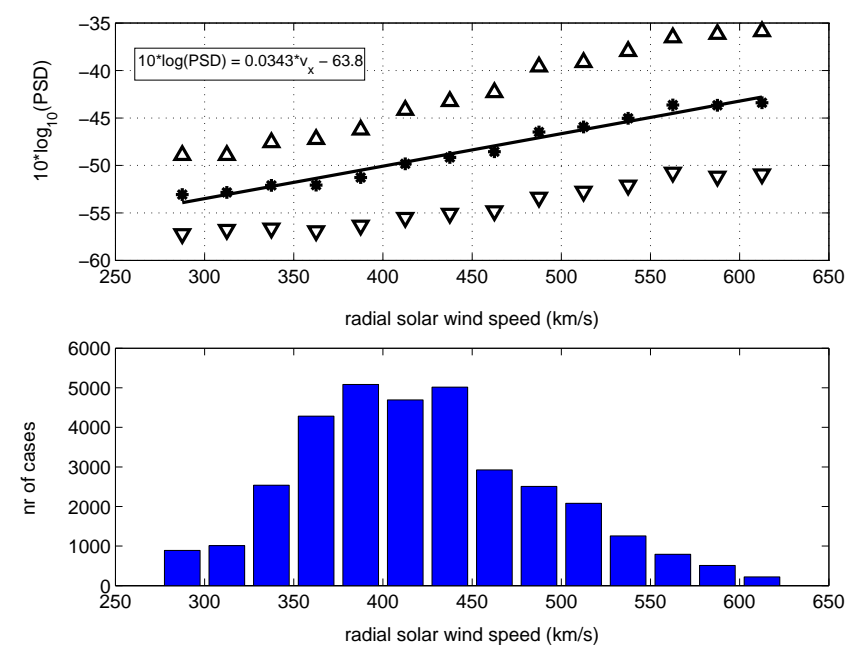

Fig. 11. Solar wind speed control of UW mean compressional power $\left(\chi<90^{\circ}\right)$. Stars represent the mean values; the solid line is the linear fit $(\mathrm{C}=0.99)$, while triangles mark the range of standard deviations. In the lower panel the solar wind speed distribution is shown.
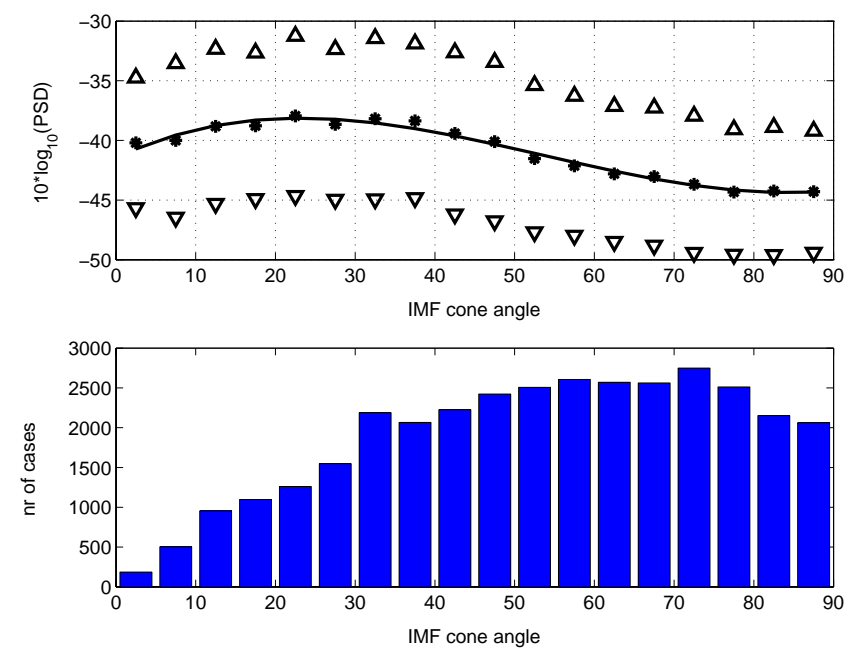

Fig. 12. Same as Fig. 11, but for IMF cone angle control of UW mean compressional power $\left(\chi<60^{\circ}\right)$.

the measurements taken at solar zenith angles $(\chi)$ smaller than $60^{\circ}$ the linear regression between power and wind speed gives $10 \cdot \log _{10}(\mathrm{PSD})=0.0343 \cdot v_{s w}-63.8$. When taking only events into account with $\chi<30^{\circ}$ the regression line has a larger slope: $10 \cdot \log _{10}(\mathrm{PSD})=0.0418 \cdot v_{s w}-65.4$. This latter expression was used in Figs. 11, 12 and 16 to normalize the compressional power for solar wind speed variations. The reference solar wind speed level was chosen to be $400 \mathrm{~km} / \mathrm{s}$.

The wave power is also controlled by the value of the cone angle (Yumoto et al., 1985). In earlier studies the maxi-

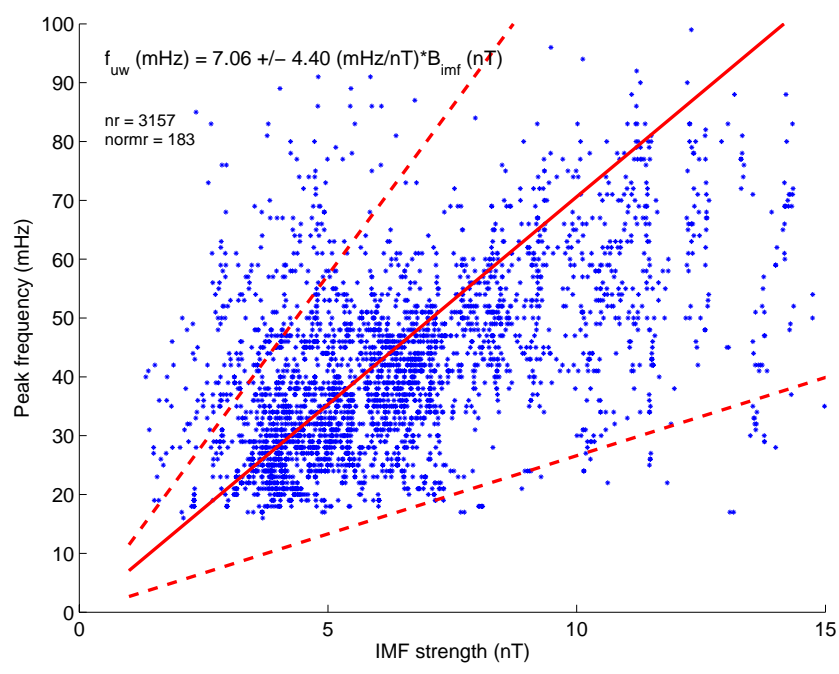

Fig. 13. Relationship between UW peak frequency and IMF strength. The solid line and the equation in the left upper corner represent the result of the linear regression; the dashed lines limit the range containing $90 \%$ of all cases.

mum was found sometimes at $30^{\circ}$ (Veró and Holló, 1978), but more frequently at $0^{\circ}$ (Wolfe et al., 1985; Yumoto et al., 1985). So far the reason for this uncertainty has not been clarified. This behaviour is a specific behaviour of UWs. During the period investigated here the maximum activity occurred at a cone angle of $22.5^{\circ}$, but the location of the peak slightly depends on the applied solar zenith angle limit.

We had already demonstrated that the local time dependence of the Group 1 activity is the same, as what is considered to be valid for UW related Pc3 pulsations in the magnetosphere and on ground. The most convincing argument for the upstream source is the dependence of the peak frequency (the frequency corresponding to the spectral peak) on the IMF strength (Fig. 13). The event selection criteria applied here were: $10 \log \mathrm{PSD}>-34, \theta_{B x}<45^{\circ}$, $-60^{\circ}<$ mag. lat. $<60^{\circ}, \chi<60^{\circ}, 9 R_{E}<R_{m p}<25 R_{E}$, where $R_{m p}$ is the subsolar distance of the magnetopause estimated by the formula $R_{m p}\left(R_{E}\right)=110 \cdot\left(n_{s w} \cdot v_{s w}^{2}\right)^{-1 / 6}$ (e.g. Kivelson and Russell, 1995, 172), and $n_{s w}$ is the number density in $\mathrm{cm}^{-3}$ and $v_{s w}$ the velocity of the solar wind plasma in $\mathrm{km} / \mathrm{s}$. This correlation clearly demonstrates the ion-cyclotron origin of this wave type. The proportionality factor of the direct correlation (7.06) is not far from the frequently cited value, $6 \mathrm{mHz} / \mathrm{nT}$ (e.g. Yumoto et al., 1984).

On the basis of all these results it may be concluded that the Group 1 activity is generated by UWs, i.e. the overwhelming majority of compressional dayside events observed in the $60^{\circ} \mathrm{N}$ to $60^{\circ} \mathrm{S}$ geomagnetic latitude range are ULF waves with upstream origin.

We cannot exclude that some UW-related activity is also present in the high-latitude regions during daytime. 
However, Group 3 activity does not follow either the cone angle dependence, or the relation between the peak frequency and the IMF strength. It means that the dominant source of the observed compressional waves in these regions cannot be the upstream wave activity.

\section{Discussion}

In this section we want to revisit the important results obtained in our extended survey and discuss possible physical mechanisms to explain them. This will be done in light of previously published results.

In Sect. 3 we have outlined the observed features of compressional Pc3-4 magnetic signals in the topside ionosphere. CHAMP, due to its high-resolution magnetometers and the good local time and latitude coverage, is particularly well suited to perform global surveys of these signatures. We have identified the compressional wave activity during daytime hours at middle latitudes as being generated by upstream waves. Many of the features which have been attributed in the past to these types of pulsations could be confirmed. The most important one is the linear relation between the peak frequency and the IMF magnitude, but also the dependence of the wave power on the solar wind speed and the modulation of the occurrence rate by the IMF clock angle. Apparent compressional wave signatures with similar frequencies but in other regions (high latitude) or in different time sectors (night time) do not exhibit the above-mentioned dependency and are not further considered in this paper.

As mentioned before, studying the UWs by means of their compressional component above the ionospheric $\mathrm{E}$ region has the advantage above ground-based observations that the signals are much cleaner. In taking advantage of this fact, several characteristics of these waves could be identified here, for the first time.

The correlation analysis between ground and space signals has also demonstrated the strong connection of subsolar compressional activity in the topside ionosphere to Pc3 activity at a mid-latitude ( $\mathrm{L}=1.83$ ) ground station. The latitudinal and longitudinal coherence lengths (THY-CHAMP) were found to be as large as 10000 and $20000 \mathrm{~km}$, and the global distribution of the average ground-space coherence is symmetric about the magnetic equator and the local magnetic meridian. Furthermore, the global distribution of THY-CHAMP and NUR-CHAMP average coherence also supports the idea that some part of the UW related ULF wave energy reaches the ground as field guided waves. However, as the UW related pulsations poleward of $+/-45^{\circ}$ mag. lat. are masked in our analysis by other magnetospheric processes, the possibility of the high-latitude (cusp?) origin of the NUR Pc3 activity cannot be excluded. The field guided propagation was proposed by Yumoto et al. (1985) in the context of FLRs, and was discussed in detail recently by Howard and Menk (2005), who emphasised the role of weakly coupled travel- ling waves in redistributing the ULF energy in the magnetosphere. As they argue, away from the resonance location where standing waves are set up, the mode conversion still occurs, but the resultant shear Alfvén waves propagate to low altitudes as field-guided travelling waves. Our case study presented in Sect. 3.1 also fits well in this picture.

Another issue is the global distribution of UWs. Some authors made an attempt to explain the low-latitude profile of magnetospheric compressional Pc3 pulsation. Kim and Takahashi (1999) suggested that the general amplitude and phase relationship between the compressional and radial component, also the ground-satellite cross-phase, could be explained consistently by standing waves in the meridian plane, which represent the plasmaspheric cavity mode. However, they could not rule out the role of incoming UWs, which in their model are coupled to evanescent mode at the plasmapause. The evanescent mode may exist inward of the turning point for fast mode compressional waves coming from the outer magnetosphere. The authors could not check the relation of their events with UWs because of the lack of IMF data. Kim and Takahashi (1999) advocated further studies to distinguish between the cavity and evanescent modes.

During the season we investigated, the September equinox, we find the highest wave power at the magnetic equator. Minima in amplitude are encountered around $40^{\circ}$ mag. lat. in both hemispheres (cf. Fig. 6). Poleward the power is increasing again. The latitudinal variation of the UW power has never been presented in this detail. Our preferred explanation for the observed shape is based on the geometry of the geomagnetic field with respect to the incoming wave front. In case the propagation vector of the compressional wave is perpendicular to the field line, the coupling should be least efficient. Conversely, the coupling is best where both are aligned and the resonance condition, $\omega_{0}=k_{\|} v_{A}$, is satisfied, where $\omega_{0}$ is the angular frequency, $k_{\|}$the parallel wave number of the driving fast mode source and $v_{A}$ the local Alfvén speed (Takahashi et al., 1994). In this way a fraction of the energy of the incoming fast mode waves locally couples to shear Alfvén mode and travels further as a guided wave along field lines. Let us assume for a first order estimate a dipolar field geometry and a plane wave front travelling from the magnetopause inward. For such a simple case the weakest coupling and highest wave power would be expected at the magnetic equator. At $\pm 35.3^{\circ}$ mag. lat. the field lines near the Earth's surface are parallel to the propagation vector of the compressional waves, thus the maximum coupling should occur further poleward of this latitude. The actual position of this maximum depends on the dominant frequency of the UW and the shape of the magnetosphere. According to the experience, field lines with 20-60 $\mathrm{mHz}$ eigenfrequency are confined under average magnetospheric conditions to a range $40^{\circ}-50^{\circ}$ mag. lat. (Menk et al., 1994). If we integrate the effect of all field line crossings up to the magnetosphere and map it to the surface, we can expect the minimum in UW related compressional wave 
activity to be more poleward. The latitudinal profile of compressional power in the topside ionosphere we presented is the result of various processes.

The UWs are confined to the dayside. This also suggests a source region close to the subsolar magnetopause. From Fig. 7, or even better, Fig. 8 we see that peak intensities are reached before noon (11:00 LT). Such an LT distribution has been reported in several earlier papers. The LT dependence of magnetospheric compressional Pc3s was first studied by Yumoto and Saito (1983), who examined the correspondence between compressional Pc3s at GOES 2 and ground Pc3 at a low-latitude $(\mathrm{L}=1.8)$ station. Simultaneous $\mathrm{Pc} 3$ events with overlapping spectral content at GOES 2 and on the ground occurred on the dayside, with the highest occurrence rate before local noon. Takahashi and Anderson (1992) carried out a statistical analysis on 4 years of AMPTE CCE $(\mathrm{L}<3)$ magnetic field data. They found that the local time distribution of the compressional wave power clearly peaks in the pre-noon sector. The pre-noon peak seems to be a general feature of both magnetospheric compressional and ground $\mathrm{Pc} 3$ pulsations. This distribution is generally attributed to the effect of the average IMF topology at the bow shock (Barnes, 1970; Greenstadt and Russell, 1994). It is believed that when the IMF orientation is close to its average direction, determined by the Parker spiral at $1 \mathrm{AU}$, i.e. when the IMF lies in the GSE XY-plane and the cone angle is about $45^{\circ}$, most of the upstream propagating ULF waves are blown back toward the morning side of the magnetosphere (Greenstadt and Russell, 1994). In this case the morning side magnetopause is quasiparallel (IMF is nearly aligned to the shock normal), and the quasi-parallel shock region is the place where UWs are generated. This is the way the cone angle influences both the LT dependence and the occurrence rate of $\mathrm{Pc} 3$ events in the magnetosphere and on the ground.

All this is in support of a partial but direct propagation of compressional waves from the magnetopause through the magnetosphere towards the Earth, as proposed, for example, by Yumoto et al. (1984). As an additional and not less important process, the mode coupling of some part of the inward propagating compressional wave energy to the shear Alfvén mode, and field guided propagation of this mode toward the ionosphere, have to be also taken into account.

\subsection{Doppler-shift of UWs}

The large scatter in the relations between ULF waves properties and solar wind parameters, as well as the often rather poor correlations, are not unusual in reports describing the solar wind/IMF control of Pc3 pulsations observed on the ground and in the magnetosphere (e.g. Verô et al., 1994). This is valid even for the best correlations that have been published (Greenstadt and Russell, 1994). In our case the scatter is further increased by some special factors. First of all, our magnetic observations in the topside ionosphere and the data of the distant ACE satellite in the interplanetary medium can- not be precisely synchronized. Our travel time calculation is only a reasonable estimation based on solar wind speed and the distance between ACE and the modelled magnetopause position. It does not account for smaller scale (in space and time) variations during the travel time. The ACE data were low-pass filtered for that reason.

Another effect which increases the scatter in the wave power in our data set is the lack of LT and latitude normalization of the observed amplitudes. As we have shown, both LT and latitude are key parameters in the study of compressional activity. Without normalization the observed signal power varies by more than a factor 5 .

Yumoto et al. (1984) tried to explain the large scatter in the relationship between the dominant Pc3 frequency and the IMF magnitude through the influence of the bulk velocity distribution of the backstreaming ion population. They developed a simple theoretical model based on the linear kinetic theory of upstream waves that may account for these observations. From the scatter of low-latitude ground and GEOS-2 magnetospheric observations, assuming that the frequency modification in the magnetosheath is small, they estimated the parallel resonant velocity of backscattered protons. Their findings $\left(v_{b}=1.6-2.7 v_{s w}\right.$ in the solar wind frame) were in acceptable agreement with the observational distribution of the reflected proton beams. Takahashi et al. (1984), following Watanabe and Terasawa (unpublished manuscript, 1983), continued this work further by developing the theoretical model, also including the effect of a possible Doppler-shift of the UWs as they are swept back by the super Alfvénic solar wind. Assuming a relationship between the guiding center velocity of backstreaming protons $\left(v_{b}\right)$ and the solar wind velocity $\left(v_{b}=2 v_{s w} / \cos \theta_{B x}\right)$, they deduced a modulation of UW frequency governed by the cone angle $\left(\omega_{s c}=1 / 2 \Omega_{p} \cos ^{2} \theta_{B x}\right.$ or $f_{s c}(\mathrm{mHz})=7.68 \cdot B_{\mathrm{IMF}}(\mathrm{nT})$ $\left.\cos ^{2} \theta_{B x}\right)$.

Later, Le and Russell (1996) revisited this problem testing the theoretical results on empirical upstream data. The resonance condition for the most unstable, right-handed magnetosonic wave is (Barnes, 1970; Yumoto et al., 1984):

$\omega_{0}-\boldsymbol{k} \cdot \boldsymbol{v}_{b}=-\Omega_{p}$,

where $\omega_{0}$ is the wave frequency in the solar wind plasma frame, $\boldsymbol{k}\left(|\boldsymbol{k}|=k_{0} \cos \theta_{k B}\right)$ is the wave vector, $\Omega_{p}$ is the proton gyrofrequency, and $v_{b}$ is the bulk (guiding center) velocity of the backstreaming ion population in the solar wind plasma frame. The reflected protons travel along the IMF and gyrorotate about the field lines, thus the UW frequency can be expressed as (Le and Russell, 1996)

$\omega_{0}=\Omega_{p} \frac{1}{\left(v_{b} / v_{p h}\right) \cos \vartheta_{k B}-1}$,

where $v_{p h}=\omega_{0} / k_{0}$ is the phase velocity of the wave, $\theta_{k B}$ is the angle between $\boldsymbol{k}$ and IMF. Supposing that the waves propagate with an Alfvén velocity $v_{p h} \approx v_{A}$ along IMF $\left(|\boldsymbol{k}|=k_{0}\right)$, 

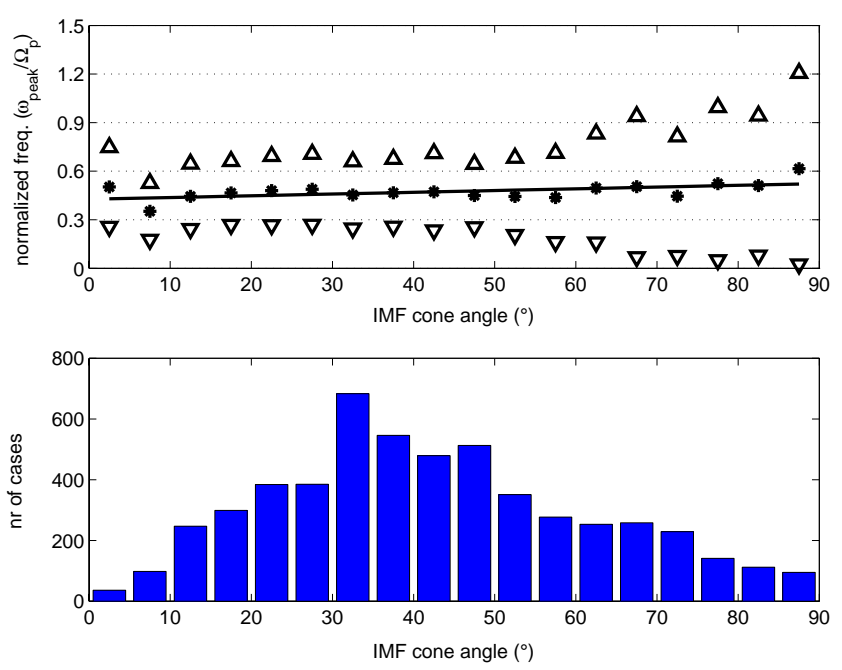

Fig. 14. The normalized UW peak frequency vs. IMF cone angle. Stars represent the mean values; the solid line is the linear fit, while triangles mark the range of standard deviations. In the lower panel the cone angle distribution is shown.

and taking into account that the solar wind is super-Alfvénic, i.e. the waves are swept downstream by the solar wind, the Doppler-shifted frequency of upstream waves in the satellite frame is given by the equation:

$\omega_{s c}=\omega_{0}+\boldsymbol{k} \cdot \boldsymbol{v}_{s w}$,

where $\boldsymbol{v}_{s w}$ is the solar wind velocity. Combining Eqs. (2) and (3) we obtain:

$\omega_{s c}=-\Omega_{p} \frac{M_{A} \cos \vartheta_{B x}-1}{M_{A} P-1}$,

which is a modified version of Eq. (5) in Takahashi et al. (1984) and that of Eq. (5) in Le and Russell (1996), where $M_{A}$ is the Alfvénic Mach number, $\theta_{B x}$ is the cone angle, $P$ is the ratio between $v_{b}$, the bulk velocity of backstreaming ions, and $v_{s w}$, the solar wind speed. In the case where the waves propagate antiparallel to the IMF, Eq. (4) alters to:

$\omega_{s c}=-\Omega_{p} \frac{M_{A} \cos \vartheta_{B x}+1}{M_{A} P+1}$,

since then $\cos \theta_{k x}$ equals $-\cos \theta_{B x}$. This later possibility, Eq. (5), was ignored in earlier studies. We note that $\omega_{s c}$ generally has a negative value. When comparing theoretical estimations (4 and 5) with CHAMP observations the absolute value of $\omega_{s c}$ will be considered. In accordance with the model sketched above, Le and Russell (1996) demonstrated by a statistical study of UW observations in the foreshock region, that the cone angle indeed plays an important role in determining the Doppler-shifted upstream ULF frequency. The normalized upstream frequency, $\omega_{s c} / \Omega_{p}$, becomes lower with increasing cone angle. The effect of solar wind Alfvénic
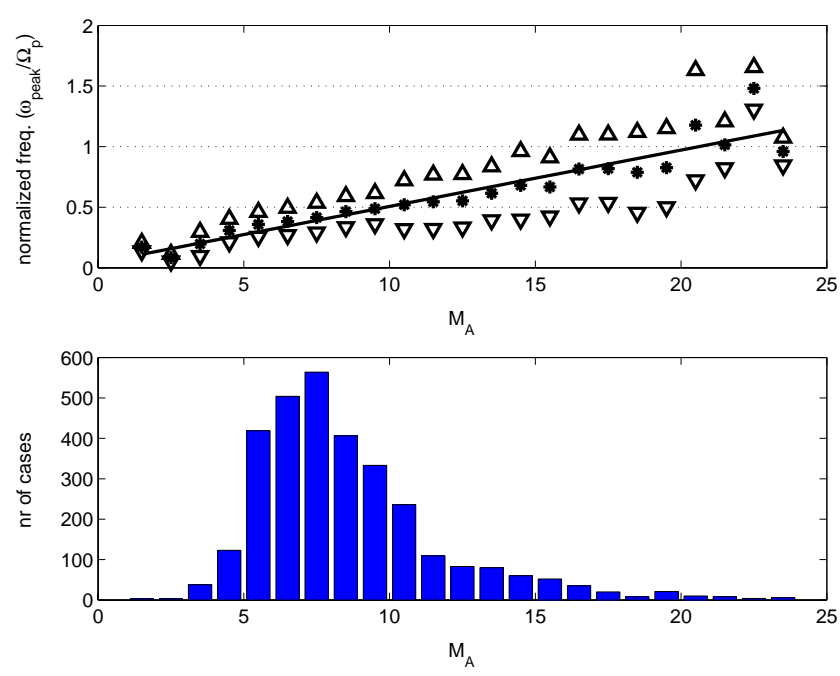

Fig. 15. Same as Fig. 14, but for normalized UW peak frequency vs. Alfvénic Mach number.

Mach number, however, was found not to have a significant influence on the frequency of the UW events detected by the ISEE spacecraft. According to the results of Le and Russell (1996), an increasing $M_{A}$ tends to enhance the UW frequency.

To check the effect of the cone angle and Alfvénic Mach number on the UW frequency we extended our statistical investigation in this direction. Our results resting on observations in the topside ionosphere are different from those of Le and Russell (1996). We found no appreciable effect of the cone angle on the normalized wave frequency, $\omega_{\text {peak }} / \Omega_{p}$ (Fig. 14, event selection criteria: $10 \log \mathrm{PSD}>-34$, $-60^{\circ}<$ mag. lat. $<60^{\circ}, \chi<60^{\circ}$, magnetopause distance is between 9 and 25 Earth radii), the distribution is fairly flat, and only the scatter increases with increasing $\theta_{B x}$.

However, consistent with theoretical predictions, a strong correlation (corr. coeff.: 0.94) between the Alfvénic Mach number and the normalized Pc3 frequency was found (Fig. 15, same events as for Fig. 13). Although this behaviour of UWs was predicted by theory, it is the first time that the Doppler shift of UWs is demonstrated convincingly based on observations. The linear regression yields the expression:

$\omega_{\text {peak }} / \Omega_{p}=0.0465 \cdot M_{A}+0.042$, or

$f_{\text {peak }}(\mathrm{mHz})=\left(0.708 \cdot M_{A}+0.64\right)(\mathrm{mHz} / \mathrm{nT}) \cdot B_{\text {imf }}(\mathrm{nT})$,

which, for the median Mach number (excluding extremes, $\left.M_{A}>25\right), M_{A}=7.75$, gives $\omega_{\text {peak }} / \Omega_{p}=0.40$. This value of normalized frequency corresponds a factor $q_{u w}=6.1 \mathrm{mHz} / \mathrm{nT}$, which is in the middle range of the values usually found by observers in the magnetosphere or on the ground. Relations (6) and (7) are the first empirical formulae which 

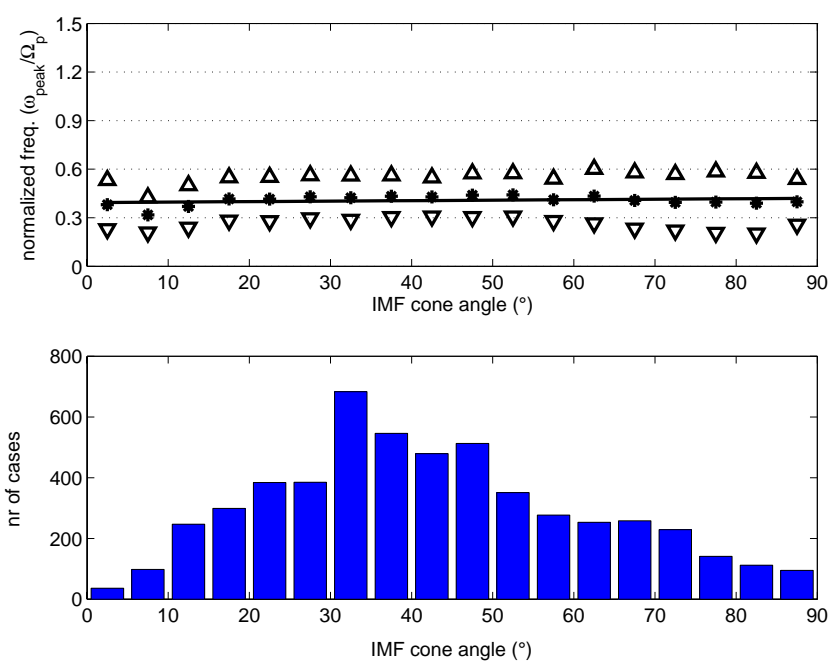

Fig. 16. Same as Fig. 14, but for corrected normalized UW peak frequency vs. IMF cone angle.

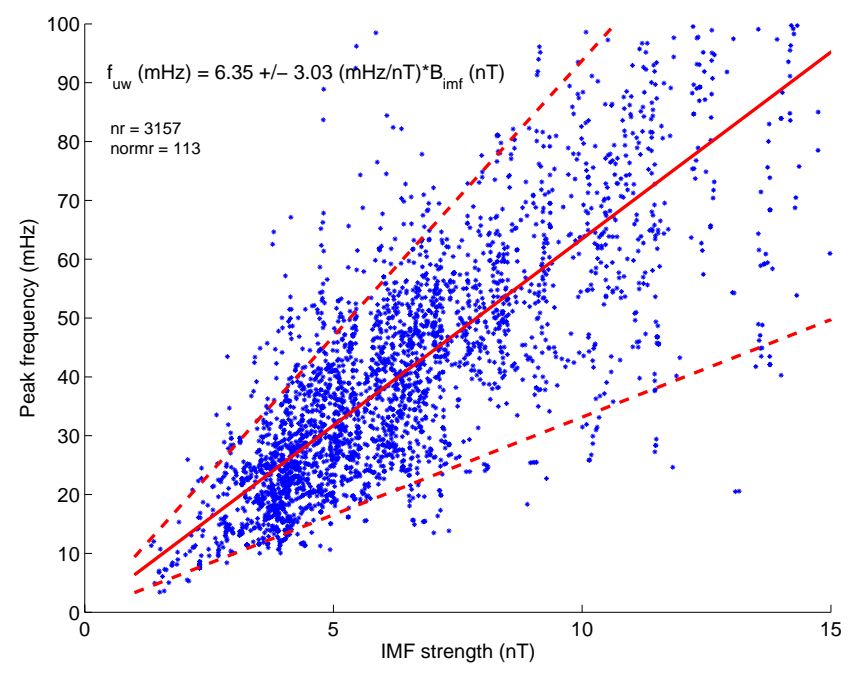

Fig. 17. Same as Fig. 13, but for corrected UW peak frequencies vs. IMF strength.

account for the Doppler shift of UWs in the foreshockmagnetosheath region. Moreover, Eq. (6) can be applied to perform a correction of the Alfvénic Mach number effect in our data. After removing the influence of $M_{A}$, the independence of the peak frequency on the cone angle becomes even more evident (Fig. 16). Furthermore, the scatter of the corrected peak frequency versus IMF field strength is significantly decreased (Fig. 17). The degree of improvement becomes visible when comparing Figs. 13 and 17.

We showed that, according to Eqs. (4) and (5), the Doppler-shifted frequency is slightly higher in the case where the UWs propagate anti-parallel to IMF, i.e. when the

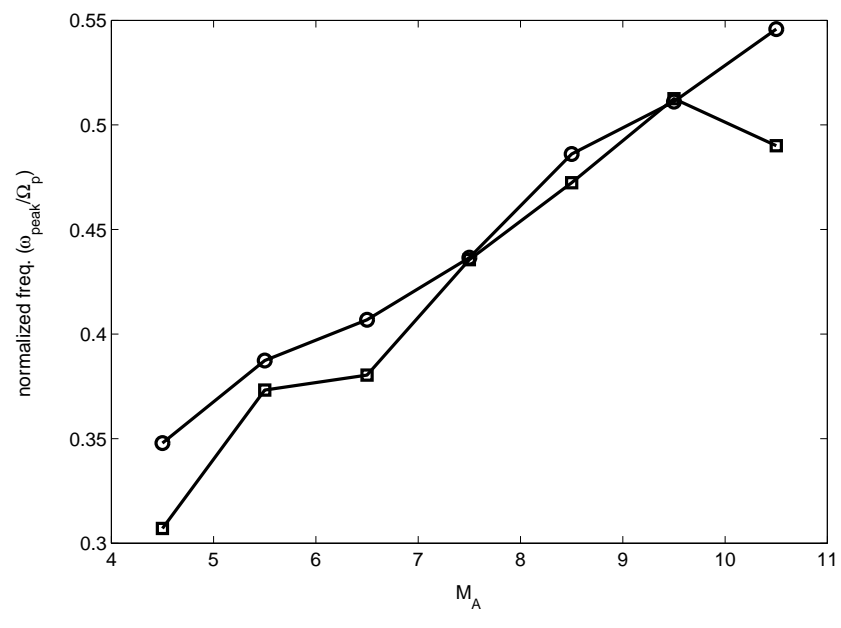

Fig. 18. The normalized UW peak frequency vs. Alfvénic Mach number. Solid lines marked by circles/rectangles represent the frequencies for inward/outward pointing IMF, respectively.

IMF points toward the Earth (inward). The predicted frequency difference in the Mach number range, $M_{A}=5-15$, assuming average values for the cone angle and $P$, is between $10-30 \%$. This effect can also be demonstrated with the CHAMP observations. The normalized frequencies are systematically higher for inward than for outward pointing IMF (Fig. 18). In the bins $M_{A}=4-5,6-7,10-11$ the differences are $13.3 \%, 7.0 \%, 11.4 \%$, respectively.

Overall, the anomalous Doppler shift of the upstream ULF wave frequency, caused by the super Alfvénic solar wind, can clearly be deduced from the compressional waves in the topside ionosphere.

\subsection{The parallel velocity of backstreaming ions}

Following Yumoto et al. (1984), and Le and Russell (1996) and considering our relationship (4) and (5) and even $P$, the ratio of $v_{b}$ over $v_{s w}$, can be estimated. Combining Eqs. (4) and (6) we can write:

$P=\frac{\Omega_{p}}{\omega_{\text {peak }}} \cos \vartheta_{B x}+\frac{1-\frac{\Omega_{p}}{\omega_{\text {peak }}}}{M_{A}}$.

Figure 19 (same events as for Fig. 13) shows the dependence of $P$ on $\cos \theta_{B x}$ (data without normalizing $\omega_{\text {peak }} / \Omega_{p}$ to average $M_{A}$ ), for which the regression analysis (corr. coeff.: $0.99)$ gives:

$P=2.48 \cdot \cos \vartheta_{B x}-0.23$.

Le and Russell (1996) obtained 2.3 as the median and 2.2 as the mean value of $P$, but could not find any significant correlation with solar wind parameters. Our result, showing the clear linear correlation between $P$ and the $\cos \theta_{B x}$ can be regarded as a direct consequence of our former result, the 
independence of the normalized frequency from the cone angle, namely, as it was demonstrated, the ratio $\omega_{\text {peak }} / \Omega_{p}$ has a median value of about 0.40 . Assuming that $\theta_{B x}$ and $M_{A}$ are independent variables (no dependence of cone angle on $M_{A}$ ) and taking into account the median value of $M_{A}=7.75$, Eq. (8) can be rewritten as:

$P=2.50 \cdot \cos \vartheta_{B x}-0.19$,

which is appreciably close to our empirical result in Eq. (9). This means that the parallel bulk speed of backscattered protons can be interpreted, on average, as the IMF aligned component of a sunward-oriented (upstream) ion speed $v_{b s}$, which is directly proportional to $v_{s w}$. Near the subsolar point of the bow shock, where the incoming protons are reflected, the shock normal is parallel to the solar wind flow, we can suppose that most of the reflected protons obtain an initial velocity $\left(\mathbf{v}_{b s}\right)$ perpendicular to the shock front, directed sunward. However, due to the IMF, these protons begin to gyrate about the field lines with a frequency $\Omega_{p}$ and travel along the IMF with a velocity $v_{b}=v_{b s} \cos \theta_{B x}$. This is in good agreement with our empirical findings. Le and Russell (1996), on the contrary, found that the normalized frequency depends on the cone angle. However, it is important to recall that their results are based on upstream measurements. In the foreshock region our above argumentation may not be valid.

\section{Summary and conclusions}

In this paper we have studied upstream wave-related compressional geomagnetic pulsations observed at LEO over four consecutive months by the CHAMP satellite.

1. We developed an algorithm for the selection of possible UW-related pulsation events from both ground and space magnetometer data, separately. The procedure uses the wavelet transformation to estimate the peak power, the integrated power and the power spectral peak or dominant frequency, as well as ground space coherence with a one-minute resolution. UW-related events are selected using a set of carefully chosen parameters (signal power, IMF cone angle, latitude, solar zenith angle).

2. This is the first mapping of the magnetic local time, as well as the magnetic latitude dependence of the compressional Pc3 power in the topside ionosphere. Our map showing the global distribution of compressional signal power in the Pc3 band at LEO altitude demonstrates the concentration of true Pc3s in the sunlit hemisphere between $60^{\circ} \mathrm{N}$ and $60^{\circ} \mathrm{S}$ mag. lat. The Pc3 power at high latitudes is masked by FAC related spatial field variations.

3. Our results support the idea of a partial but direct propagation of compressional waves from the subsolar
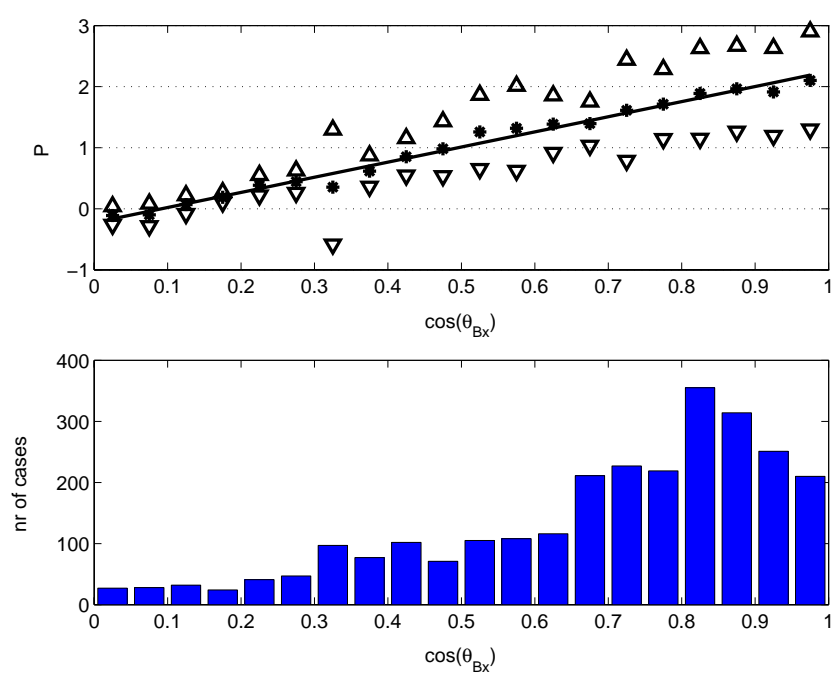

Fig. 19. The ratio of bulk velocity of back-streaming protons over solar wind velocity, $P$ vs. cosine of the IMF cone angle. Stars represent the mean values; the solid line is the linear fit, while triangles mark the range of standard deviations. In the lower panel the cosine cone angle distribution is shown.

magnetopause through the magnetosphere towards the Earth, as proposed by Yumoto et al. (1984). At the same time, a channel which is no less important, UW waves guided along local field lines, must also be taken into account, as suggested by Howard and Menk (2005). Some part of the inward propagating compressional wave energy weakly couples to field guided shear Alfvén mode, playing an important role in redistributing the ULF energy in the magnetosphere.

4. One of the most important results of this survey is the first observational verification of the Doppler shift of UWs by the solar wind, as predicted by theory (Le and Russell, 1996). It is shown that the UW frequency, which is controlled primarily by the IMF strength, strongly depends on the Alfvénic Mach number in the upstream region. On the other hand, the demonstrated independence of the UW frequency on the cone angle implies that the parallel speed of backstreaming ions is determined not only by the solar wind speed but also by the cone angle.

In a next step we will extend our analysis to a longer period to be able to study the seasonal variation of the latitudinal and local time distribution of Pc3 power. Our results presented here are valid for equinox conditions. We also started a ground-space cross-phase analysis that yields information on the direction of wave propagation in the topside ionosphere. The results of this analysis are not presented here. Radial and toroidal Pc3 pulsation components were not treated in this paper. The investigation of mode coupling, 
however, will demand the inclusion of all components in future studies.

As it was mentioned in the Introduction, upstream ULF waves can also enter the magnetosphere directly at the cusp. Further studies are needed to develop methods that are suitable for identifying UW-related Pc3s from satellite observations at high latitudes.

Multi-site case studies, analysing simultaneous space data from the upstream region, the magnetosheath, the magnetosphere, the topside ionosphere and also from ground magnetometer arrays, are needed to obtain a complete view of the whole process. Our results show that low- and mid-latitude ground stations have the advantage opposite to high-latitude stations that the Pc3 activity here has a more direct connection to the ULF waves in the magnetosphere. In the study of UW-related pulsations these stations, and especially low and mid latitude magnetometer arrays, will play a considerable role.

Acknowledgements. The authors are grateful to J. Veró and P. Bencze for helpful discussions. A major part of this study was performed during a research visit of B. Heilig at GFZ Potsdam. We thank L. Pankratz, E. Sauter (USGS, U.S.), J. Kultima, T. Raita (SGO, Finnland), K. Pajunpää (FMI, Finnland), J. Reda (PAS, Poland), M. Váczyová, Z. Vörös (SAS, Slovakia), V. Wesztergom (HAS GGRI, Hungary), A. Csontos, L. Szabados (ELGI, Hungary) and to their institutes for maintaining the MM100 magnetometer array. The provision of the ACE data by the ACE MAG and SWEPAM instrument teams and the ACE Science Center greately acknowledged. This study is supported by the Hungarian Space Office (TP 153) and by the Hungarian Scientific Research Fund (NI 61013).

Topical Editor I. A. Daglis thanks K. Takahashi and A. Belehaki for their help in evaluating this paper.

\section{References}

Araki, T.: A physical model of the geomagnetic sudden commencement, in: Solar wind sources of magnetospheric ultra-lowfrequency waves, pp. 183-200, Geophysical Monogr. 81, AGU, Washington, D.C., 1994.

Barnes, A.: Theory of generation of bow-shock-associated hydromagnetic waves in the upstream interplanetary medium, Cosmic Electrodyn., 1, 90-97, 1970.

Bol'shakova, O. V. and Troitskaya, V.: Relation of the IMF direction to the system of stable oscillations, Doklady Akademii Nauk (Proceedings of the Russian Academy of Sciences), 180, 343 346, 1968.

Chen, L. and Hasegawa, A.: A theory of long-period magnetic pulsations, 1. Steady state excitation of field line resonance, J. Geophys. Res., 79, 1024-1032, 1974.

Dungey, J. W.: Electrodynamics of the outer atmosphere: report to National Science Foundation on work carried on under grant NSF-G450, Pennsylvania State University, Ionosphere Research Laboratory, University Park, 1954

Engebretson, M. J., Cahill, L. J., R. L., Jr., A., Anderson, B. J., Rosenberg, T. J., Carpenter, D. L., Inan, U. S., and Eather,
R. H.: The role of the ionosphere in coupling upstream ULF wave power into the dayside magnetosphere, J. Geophys. Res., 96, 1527-1542, 1991a.

Engebretson, M. J., Lin, N., Baumjohann, W., Lühr, H., Anderson, B. J., Zanetti, L. J., Potemra, T. A., McPherron, R. L., and Kivelson, M. G.: A comparison of ULF fluctuations in the solar wind, magnetosheath, and dayside magnetosphere. 1. Magnetosheath morphology, J. Geophys. Res., 96, 3441-3454, 1991b.

Gary, S. P.: Electromagnetic ion beam instability and energy loss of fast alpha particles, Nucl. Fusion, 18, 327-334, 1978.

Gary, S. P.: Electromagnetic ion ion instabilities and their consequences in space plasmas - a review, Space Sci. Rev., 56, 373 415, 1991.

Green, C. A., Odera, T. J., and Stuart, W. F.: The relationship between the strength of the IMF and the frequency of magnetic pulsations on the ground and in the solar wind, Planet. Space Sci., 31, 559-567, 1983.

Greenstadt, E. W. and Olson, J. V.: A contribution to ULF activity in the Pc 3-4 range correlated with IMF radial orientation, J. Geophys. Res., 82, 4991-4996, 1977.

Greenstadt, E. W. and Russell, C. T.: Stimulation of exogenic, daytime geomagnetic pulsations: a global perspective, in: Solar wind sources of magnetospheric ultra-low-frequency waves, pp. 13-23, Geophysical Monogr. 81, AGU, Washington, D.C., 1994.

Grinsted, A., Moore, J. C., and Jevrejeva, S.: Application of the cross wavelet transform and wavelet coherence to geophysical time series, Nonlin. Processes Geophys., 11, 561-566, 2004, http://www.nonlin-processes-geophys.net/11/561/2004/.

Gul'elmi, A. V., Plyasova-Bakunina, T. A., and Shchepetnov, R. V.: Relation between the period of geomagnetic pulsations Pc 3, 4 and the parameters of the interplanetary medium at Earth's orbit, Geomagn. Aeron., 13, 331-334, 1973.

Howard, T. A. and Menk, F. W.: Ground observations of highlatitude Pc3-4 ULF waves, J. Geophys. Res., 110, A04 205, doi:10.1029/2004JA010417, 2005.

Hughes, W. J.: Magnetospheric ULF waves: A tutorial with a historical perspective, in: Solar wind sources of magnetospheric ultra-low-frequency waves, pp. 1-11, Geophysical Monogr. 81, AGU, Washington, D.C., 1994.

Hughes, W. J., McPherron, R. L., and Barfield, J. N.: Geomagnetic pulsations observed simultaneously on three geostationary satellites, J. Geophys. Res., 83, 1109-1116, 1978.

Jadhav, G., Rajaram, M., and Rajaram, R.: Modification of daytime compressional waves by the ionosphere: first results from Oersted, Geophys. Res. Lett., 28, 103-106, 2001.

Kim, K.-H. and Takahashi, K.: Statistical analysis of compressional Pc3-4 pulsations observed by AMPTE CCE at L $=2-3$ in the dayside magnetosphere, J. Geophys. Res., 104, 4539-4558, 1999.

Kim, K.-H., Takahashi, K., and Anderson, B. J.: Ground-satellite coherence analysis of Pc3 pulsations, J. Geophys. Res., 103, 11755-11 769, 1998.

Kivelson, M. G. and Russell, C. T.: Introduction to space physics, Cambridge University Press, London, 1995.

Kivelson, M. G. and Southwood, D. J.: Resonant ULF waves: A new interpretation, Geophys. Res. Lett., 12, 49-52, 1985.

Kivelson, M. G. and Southwood, D. J.: Coupling of global magnetospheric MHD eigenmodes to field line resonances, J. Geophys. 
Res., 91, 4345-4351, 1986.

Kovner, M. S., Lebedev, V. V., Plyasova-Bakunina, T. A., and Troitskaya, V. A.: On the generation of low frequency waves in the solar wind in front of the bow shock, Planet. Space Sci., 24, 261267, 1976.

Krauss-Varban, D.: Bow shock and magnetosheath simulations: wave transport and kinetic properties, in: Solar wind sources of magnetospheric ultra-low-frequency waves, pp. 121-134, Geophysical Monogr. 81, AGU, Washington, D.C., 1994.

Le, G. and Russell, C. T.: The morphology of ULF waves in the Earth's foreshock, in: Solar wind sources of magnetospheric ultra-low-frequency waves, pp. 87-98, Geophysical Monogr. 81, AGU, Washington, D.C., 1994.

Le, G. and Russell, C. T.: Solar wind control of upstream wave frequency, J. Geophys. Res., 101, 2571-2575, 1996.

Lühr, H., Aylward, A., Buchert, S. C., Pajunpää, A., Pajunpää, K., Holmboe, T., and Zalewski, S. M.: Westward moving dynamic substorm features observed with the IMAGE magnetometer network and other ground-based instruments, Ann. Geophys., 16, 425-440, 1998,

http://www.ann-geophys.net/16/425/1998/.

Lühr, H., Maus, S., Rother, M., and Cooke, D.: First in-situ observation of night time F-region currents with the CHAMP satellite, Geophys. Res. Lett., 29(10), 1489, doi:10.1029/2001GL013845, 2002.

Maus, S., Lühr, H., Balasis, G., Rother, M., and Mandea, M.: Introducing POMME, The Potsdam Magnetic Model of the Earth, in: Earth Observation with CHAMP, edited by: Reigber, C., Lühr, H., Schwintzer, P., and Wickert, J., pp. 293-298, Springer, Berlin-Heidelberg, 2005.

Maus, S., Rother, M., Hemant, K., Stolle, C., Lühr, H., Kuvshinov, A., and Olsen, N.: Earth's lithospheric magnetic field determined to spherical harmonic degree 90 from CHAMP satellite measurements, Geophys. J. Int., 164(2), 319-330, doi:10.1111/j.1365-246X.2005.02833.x, 2006.

Menk, F. W., Fraser, B. J., Waters, C. L., Ziesolleck, C. W. S., Feng, Q., Lee, S. H., and McNabb, P. W.: Ground measurements of low latitude magnetospheric field line resonances, in: Solar wind sources of magnetospheric ultra-low-frequency waves, pp. 299310, Geophysical Monogr. 81, AGU, Washington, D.C., 1994.

Nishida, A.: Geomagnetic diagnosis of the magnetosphere, Springer, New York, 1978.

Olson, J. V. and Fraser, B. J.: Pc3 pulsations in the cusp, in: Solar wind sources of magnetospheric ultra-low-frequency waves, pp. 325-334, Geophysical Monogr. 81, AGU, Washington, D.C., 1994.

Olson, J. V. and Rostoker, G.: Longitudinal phase variations of Pc 4-5 micropulsations, J. Geophys. Res., 83, 2481-2488, 1978.

Plyasova-Bakunina, T. A.: Effect of IMF on the characteristics of Pc 2-4 pulsations, Geomag. Aeron., 12, 135-145, 1972.

Popatov, A. S. and Mazur, V. A.: Pc3 pulsations: from the source in the upstream region to Alfvén resonances in the magnetosphere. Theory and observations, in: Solar wind sources of magnetospheric ultra-low-frequency waves, pp. 325-334, Geophysical Monogr. 81, AGU, Washington, D.C., 1994.

Russell, C. T. and Hoppe, M. M.: The dependence of upstream wave periods on the interplanetary magnetic field strength, Geophys. Res. Lett., 8, 615-618, 1981.

Russell, C. T., Luhmann, J. G., Odera, T. J., and Stuart, W. F.: The rate of occurrence of dayside Pc 3,4 pulsations: The L-value dependence of the IMF cone angle effect, Geophys. Res. Lett., 10, 663-666, 1983.

Saito, T.: A new index of geomagnetic pulsation and its relation to solar M-regions, Rept., Ionosph. Space Res., Japan, 18, 260-274, 1964.

Samson, J. C. and Rankin, R.: The coupling of solar wind energy to MHD cavity modes, waveguide modes, and field line resonances in the Earth's Magnetosphere, in: Solar wind sources of magnetospheric ultra-low-frequency waves, pp. 253-264, Geophysical Monogr. 81, AGU, Washington, D.C., 1994.

Samson, J. C., Harrold, B. H., Ruohoniemi, J. M., Greenwald, R. A., and Walker, A. D. M.: Field line resonances associated with MHD waveguides in the magnetosphere, Geophys. Res. Lett., 19, 441-444, 1992.

Singer, H. J., Russell, C. T., Kivelson, M. G., Greenstadt, E. W., and Olson, J. V.: Evidence for control of Pc 3,4 magnetic pulsations by solar wind velocity, Geophys. Res. Lett., 4, 377-380, 1977.

Southwood, D. J.: Some features of field line resonances in the magnetosphere, Planet. Space Sci., 22, 483-491, 1974.

Stolle, C., Lühr, H., Rother, M., and Balasis, G.: Magnetic signatures of equatorial spread $\mathrm{F}$, as observed by the CHAMP satellite, J. Geophys. Res., 111, A02 304, doi:10.1029/2005JA011184, 2006.

Sutcliffe, P. R. and Lühr, H.: A comparison of Pi2 pulsations observed by CHAMP in low Earth orbit and on the ground at low latitude, Geophys. Res. Lett., 30(21), 2105, doi:10.1029/2003GL018270, 2003.

Takahashi, K. and Anderson, B. J.: Distribution of ULF energy ( $\mathrm{f}<80 \mathrm{mHz}$ ) in the inner magnetosphere: a statistical analysis of AMPTE CCE magnetic field data, J. Geophys. Res., 97, 10751$10773,1992$.

Takahashi, K., McPherron, R. L., and Terasawa, T.: Dependence of the spectrum of Pc 3-4 pulsations on the interplanetary magnetic field, J. Geophys. Res., 89, 2770-2780, 1984.

Takahashi, K., Anderson, B. J., and Yumoto, K.: Upper atmosphere research satellite observation of a Pi2 pulsation, J. Geophys. Res., 104, 25 035-25 045, 1999.

Takahashi, K. B., Anderson, B. J., Newell, P. T., Yamamoto, T., and Sato, N.: Propagation of compressional Pc3 pulsations from space to the ground: a case study using multipoint measurements, in: Solar wind sources of magnetospheric ultra-lowfrequency waves, pp. 355-363, Geophysical Monogr. 81, AGU, Washington, D.C., 1994.

Tanaka, Y.-M., Yumoto, K., Shinohara, M., Kitamura, T.-I., Solovyev, S. I., Vershinin, E. F., Fraser, B. J., and Cole, D.: Coherent Pc 3 pulsations in the prenoon sector observed along the $210^{\circ}$ magnetic meridian, Geophys. Res. Lett., 25, 3477-3480, 1998.

Tanaka, Y.-M., Yumoto, K., Yoshikawa, A., Shinohara, M., Kawano, H., and Kitamura, T.-I.: Longitudinal structure of Pc3 pulsations on the ground near the magnetic equator, J. Geophys. Res., 109, A03 201, doi:10.1029/2003JA009903, 2004.

Troitskaya, V. A.: Discoveries of sources of Pc2-4 waves - A Review of research int he former USSR, in: Solar wind sources of magnetospheric ultra-low-frequency waves, pp. 45-54, Geophysical Monogr. 81, AGU, Washington, D.C., 1994.

Troitskaya, V. A. and Bol'shakova, O. V.: The relation of the high latitude maximum of $\mathrm{Pc} 3$ intensity to the dayside cusp, Geo- 
magn. Aeron., 7, 633-635, 1984.

Troitskaya, V. A., Plyasova-Bakounina, T. A., and Gul'elmi, A. V.: Relationship between Pc 2-4 pulsations and the interplanetary magnetic field (in Russian), Doklady Akademii Nauk, Proceedings of the Russian Academy of Sciences, 197, 1312-1314, 1971.

Vellante, M., Lühr, H., Zhang, T. L., Wesztergom, V., Villante, U., Lauretis, M. D., Piancatelli, A., Rother, M., Schwiegenschuh, K., Koren, W., and Magnes, W.: Ground/satellite Signatures of Field Line Resonance: A test of theoretical predictions, J. Geophys. Res., 109, A06 210, doi:10.1029/2004JA010392, 2004.

Verő, J.: Geomagnetic pulsations and parameters of the interplanetary medium, J. Atmos. Terr. Phys., 42, 371-380, 1980.

Verô, J.: Experimental aspects of low-latitude pulsations - a review, J. Geophys., 60, 106-120, 1986.

Verô, J. and Holló, L.: Connection between interplanetary magnetic field and geomagnetic pulsations, J. Atmos. Terr. Phys., 40, 857 865,1978

Verő, J. and Zieger, B.: Upstream waves and field line resonances pulsation research at the Nagycenk observatory during three solar cycles, in: Solar wind sources of magnetospheric ultra-lowfrequency waves, pp. 55-66, Geophysical Monogr. 81, AGU, Washington, D.C., 1994.

Verő, J., Zieger, B., and Lühr, H.: Upstream waves and surface geomagnetic pulsations, in: Solar wind sources of magnetospheric ultra-low-frequency waves, pp. 365-373, Geophysical Monogr., 81, AGU, Washington, D.C., 1994.

Wang, H., Lühr, H., and Ma, S. Y.: Solar zenith angle and merging electric field control of field-aligned currents: A statistical study of the Southern Hemisphere, J. Geophys. Res., 110, A03 306, doi:10.1029/2004JA010530, 2005.
Waters, C. L., Takahashi, K., Lee, D.-H., and Anderson, B. J.: Detection of ultralow-frequency cavity modes using spacecraft data, J. Geophys. Res., 107, 1284, doi:10.1029/2001JA000224, 2002.

Wolfe, A.: Dependence of mid-latitude hydromagnetic energy spectra on solar wind speed and interplanetary magnetic field direction, J. Geophys. Res., 85, 5977-5982, 1980.

Wolfe, A., Meloni, A., Lanzerotti, L. J., and MacLennan, C. G.: Dependence of hydromagnetic energy spectra near $\mathrm{L}=2$ and $\mathrm{L}=3$ on upstream solar wind parameters, J. Geophys. Res., 90, 51175131, 1985.

Wright, A. N.: Dispersion and wave coupling in inhomogeneous MHD waveguides, J. Geophys. Res., 99, 159-167, 1994.

Yedidia, B. A., Vellante, M., Villante, U., and Lazarus, A. J.: A study of the relationship between micropulsations and solar wind properties, J. Geophys. Res., 96, 3465-3470, 1991.

Yumoto, K. and Saito, T.: Relation of compressional HM waves at GOES 2 to low-latitude Pc 3 magnetic pulsations, J. Geophys. Res., 88, 10 041-10 052, 1983.

Yumoto, K., Saito, T., Tsurutani, B. T., Smith, E. J., and Akasofu, S.-I.: Relationship between the IMF magnitude and Pc 3 magnetic pulsations in the magnetosphere, J. Geophys. Res., 89, 9731-9740, 1984.

Yumoto, K., Saito, T., Akasofu, S.-I., Tsurutani, B. T., and Smith, E. J.: Propagation mechanism of daytime Pc 3-4 pulsations observed at synchronous orbit and multiple ground-based stations, J. Geophys. Res., 90, 6439-6450, 1985. 